\title{
Light scattering of Laguerre-Gaussian beams: near-field structures and symmetries
}

\author{
A. D. Kiselev ${ }^{1}$, D. O. Plutenko ${ }^{2,3}$ \\ ${ }^{1}$ ITMO University, Kronverkskiy, 49, St. Petersburg, 197101, Russia \\ ${ }^{2}$ Institute of Physics of National Academy of Sciences of Ukraine, Kiev, Ukraine \\ ${ }^{3}$ Physical Engineering Teaching Research Center of National Academy of Sciences of Ukraine, \\ Kiev, Ukraine \\ alexei.d.kiselev@gmail.com,dmplutenko@gmail.com
}

PACS 42.25.Fx, 42.68.Mj, 42.25.Bs

DOI 10.17586/2220-8054-2016-7-2-349-370

\begin{abstract}
We apply the method of far-field matching to remodel laser beams and study light scattering from spherical particles illuminated by a Laguerre-Gaussian (LG) light beam. The optical field in the near-field region is analyzed for purely azimuthal LG beams characterized by a nonzero azimuthal mode number $m_{\mathrm{LG}}$. The morphology of photonic nanojets is shown to significantly vary, depending the mode number and the scatterer's characteristics. The cases of negative index metamaterial and metallic Mie scatterers are discussed. We also discuss the symmetry properties of laser beams and related results for the optical forces. The near-field structure of optical vortices associated with the components of the electric field, being highly sensitive to the mode number, is found to be determined by the twofold rotational symmetry.
\end{abstract}

Keywords: light scattering, Laguerre-Gaussian beams, photonic nanojets, optical vortices.

Received: 22 January 2016

\section{Introduction}

The scattering of light and other radiation by particles has long been known to be of crucial importance in a great variety of science and engineering disciplines. The problem of light scattering by spherically shaped particles dates back to the more than century-old classical exact solution due to Mie [1]. The analysis of a Mie-type theory uses a systematic expansion of the electromagnetic field over vector spherical harmonics [2-5]. The specific form of the expansions is also known as the $T$-matrix ansatz that has been widely used in the related problem of light scattering by nonspherical particles $[4,6,7]$. More recently, this strategy has been successfully applied to optically anisotropic particles [8-13].

The Mie solution, in its original form, applies to the scattering of plane electromagnetic waves by uniform optically isotropic spherical particles (the so-called Mie scatterers). For laser beams, it is generally necessary to go beyond the plane-wave approximation and light scattering from arbitrary shaped laser beams [14-18] has been the key subject of the Mietype theory - the so-called generalized Lorenz-Mie theory (GLMT) - extended to the case of arbitrary incident-beam scattering $[5,19]$. In such generalization of the Mie theory, the central and the most important task is to describe the illuminating beams in terms of expansions over a set of basis wavefunctions. In GLMT, a variety of methods were developed to evaluate the expansion coefficients that are referred to as the beam shape coefficients (for a recent review see Ref. [20] and references therein).

The central problem with laser beams is due to the fact that in their standard mathematical form, these beams are not radiation fields which are solutions to Maxwell's equations. 
Typically, the analytical treatment of laser beams is performed using the paraxial approximation [21] and the beams are described as pseudo-fields which are only approximate solutions of the vector Helmholtz equation (higher order corrections can be used to improve the accuracy of the paraxial approximation $[21,22])$.

Unfortunately, multipole expansions do not exist for such approximate pseudo-fields. Therefore, some remodelling procedure must be invoked to obtain a real radiation field which can be regarded as an approximation to the original paraxial beam.

The basic concept that might be called matching the fields on a surface lies at the heart of various traditional approaches to the laser beam remodelling and is based on the assumption that there is a surface where the actual incident field is equal to the paraxial field. Examples of physically reasonable and natural choice are scatterer-independent matching surfaces such as a far-field sphere [23], the focal plane (for beams with well-defined focal planes) [23,24], and a Gaussian reference sphere representing a lens [25]. Given the paraxial field distribution on the matching surface, the beam shape coefficients can be evaluated using either numerical integration or the one-point matching method [23].

An alternative approach is to analytically describing the propagation of a laser beam, which is known in the paraxial limit, without recourse to the paraxial approximation. In Refs. [26-30] this strategy has been applied to the important case of Laguerre-Gaussian (LG) beams using different methods such as the vectorial Rayleigh-Sommerfeld formulas [27,30], the vector angular spectrum method [29], approximating LG beams by nonparaxial beams with (near) cylindrical symmetry [26,28].

The nonparaxial beams are solutions of Maxwell's equations and the beam shape coefficients can be computed using the methods of GLMT. In recent studies of light scattering by spherical and spheroidal particles illuminated with LG beams [31,32], the analytical results of Ref. [28] were used to calculate the beam shape coefficients.

In this paper, the problem of light scattering from LG beams that represent optical vortex laser beams exhibiting a helical phase front and carrying a phase singularity will be of our primary interest. The topological charge characterizing the phase singularity and associated orbital angular momentum gives rise to distinctive phenomena such as soliton generation [33], entanglement of photon quantum states, orbital angular momentum exchange with atoms and molecules (in addition to the collection of papers [34], see reviews in Ref. [35]), rotation and orbital motion of spherical particles illuminated with LG beams [36,37].

In our calculations, we shall follow Refs. [10,38] and use the $T$-matrix approach in which the far-field matching method is combined with the results for nonparaxial propagation of LG beams $[29,30]$. Our goal is to examine the near-field structure of optical field depending on the parameters characterizing both the beam and the scatterer.

This structure has recently attracted considerable attention that was stimulated by an upsurge of interest in the so-called photonic nanojets and their applications (for a review see Ref. [39]). These nanojets were originally identified in finite-difference-time-domain simulations $[40,41]$ as narrow, high-intensity electromagnetic beams that propagate into background medium from the shadow-side surface of a plane-wave illuminated dielectric microcylinder [40] or microsphere [41] of diameter greater than the illuminating wavelength. There are several potentially important applications for the photonic nanojets to detect and manipulate nanoscale objects, subdiffraction-resolution nanopattering and nanolithography, low-loss waveguiding, and ultra-density optical storage. These applications are reviewed in Ref. [39].

The layout of the paper is as follows: in Sec. 2, we outline our theoretical approach. The analytical results for the beam shape coefficients of LG beams and the fundamental properties of the far-field angular distributions are described in Sec. 3. The numerical procedure and the 
results of numerical computations representing the near-field intensity distributions and phase maps of electric field components for purely azimuthal LG beams are presented in Sec. 4 . Finally, in Sec. 5, we present our results and make some concluding remarks.

\section{Lorenz-Mie theory: Wave functions and $\boldsymbol{T}$-matrix}

In this section, we introduce all necessary notations and briefly discuss how the properties of Mie scattering can be described in terms of the $T$-matrix [2,4]. Our formulation closely follows to the line of our presentation given in Refs. [10,38].

We consider scattering by a spherical particle of radius $R_{p}$ embedded in a uniform isotropic dielectric medium with dielectric constant $\epsilon_{\text {med }}$ and magnetic permeability $\mu_{\text {med }}$. The dielectric constant and magnetic permittivity of the particle are $\epsilon_{p}$ and $\mu_{p}$, respectively. For a harmonic electromagnetic wave (time-dependent factor is $\exp \{-i \omega t\}$ ), the Maxwell equations can be written in the following form:

$$
\begin{aligned}
-i k_{i}^{-1} \nabla \times \mathbf{E} & =\frac{\mu_{i}}{n_{i}} \mathbf{H}, \\
i k_{i}^{-1} \nabla \times \mathbf{H} & =\frac{n_{i}}{\mu_{i}} \mathbf{E}, \quad i= \begin{cases}\operatorname{med}, & r>R_{p} \\
p, & r<R_{p}\end{cases}
\end{aligned}
$$

where $n_{\text {med }}=\sqrt{\epsilon_{\text {med }} \mu_{\text {med }}}$ is the refractive index outside the scatterer (in the ambient medium), where $r>R_{p}(i=$ med $)$ and $k_{i}=k_{\text {med }}=n_{\text {med }} k_{\text {vac }}\left(k_{\mathrm{vac}}=\omega / c=2 \pi / \lambda\right.$ is the free-space wave number); $n_{p}=\sqrt{\epsilon_{p} \mu_{p}}$ is the refractive index for the region inside the spherical particle (scatterer), where $r<R_{p}(i=p)$ and $k_{i}=k_{p}=n_{p} k_{\mathrm{vac}}$.

The electromagnetic field can always be expanded using the vector spherical harmonic basis [42]. There are three cases of these expansions that are of particular interest. They correspond to the incident wave, $\left\{\mathbf{E}_{\text {inc }}, \mathbf{H}_{\text {inc }}\right\}$, the outgoing scattered wave, $\left\{\mathbf{E}_{\text {sca }}, \mathbf{H}_{\text {sca }}\right\}$ and the electromagnetic field inside the scatterer, $\left\{\mathbf{E}_{p}, \mathbf{H}_{p}\right\}$ :

$$
\begin{aligned}
& \mathbf{E}_{\alpha}=\sum_{j m}\left[\alpha_{j m}^{(\alpha)} \mathbf{M}_{j m}^{(\alpha)}\left(\rho_{i}, \hat{\mathbf{r}}\right)+\beta_{j m}^{(\alpha)} \mathbf{N}_{j m}^{(\alpha)}\left(\rho_{i}, \hat{\mathbf{r}}\right)\right], \quad \alpha \in\{\text { inc, sca, } p\} \\
& \mathbf{H}_{\alpha}=n_{i} / \mu_{i} \sum_{j m}\left[\alpha_{j m}^{(\alpha)} \mathbf{N}_{j m}^{(\alpha)}\left(\rho_{i}, \hat{\mathbf{r}}\right)-\beta_{j m}^{(\alpha)} \mathbf{M}_{j m}^{(\alpha)}\left(\rho_{i}, \hat{\mathbf{r}}\right)\right], \\
& \mathbf{M}_{j m}^{(\alpha)}\left(\rho_{i}, \hat{\mathbf{r}}\right)=i k_{i}^{-1} \nabla \times \mathbf{N}_{j m}^{(\alpha)}=z_{j}^{(\alpha)}\left(\rho_{i}\right) \mathbf{Y}_{j m}^{(m)}(\hat{\mathbf{r}}), \\
& \mathbf{N}_{j m}^{(\alpha)}\left(\rho_{i}, \hat{\mathbf{r}}\right)=-i k_{i}^{-1} \nabla \times \mathbf{M}_{j m}^{(\alpha)}=\frac{\sqrt{j(j+1)}}{\rho_{i}} z_{j}^{(\alpha)}\left(\rho_{i}\right) \mathbf{Y}_{j m}^{(0)}(\hat{\mathbf{r}})+D z_{j}^{(\alpha)}\left(\rho_{i}\right) \mathbf{Y}_{j m}^{(e)}(\hat{\mathbf{r}}), \\
& i=\left\{\begin{array}{ll}
\text { med, } & \alpha \in\{\text { inc }, \text { sca }\} \\
p, & \alpha=p
\end{array}, \quad z_{j}^{(\alpha)}\left(\rho_{i}\right)= \begin{cases}j_{j}(\rho), & \alpha=\text { inc } \\
h_{j}^{(1)}(\rho), & \alpha=\text { sca }, \\
j_{j}\left(\rho_{p}\right), & \alpha=p\end{cases} \right.
\end{aligned}
$$

where $\rho \equiv \rho_{\text {med }}=k_{\text {med }} r, \rho_{p}=k_{p} r \equiv n \rho$, and $n=n_{p} / n_{\text {med }}$ is the ratio of refractive indexes also known as the optical contrast; $D f(x) \equiv x^{-1} \partial_{x}(x f(x))$. 
According to Ref. [10], the spherical harmonics can be conveniently expressed in terms of the Wigner $D$-functions $[42,43]$ as follows:

$$
\begin{aligned}
& \mathbf{Y}_{j m}^{(m)}(\hat{\mathbf{r}})=N_{j} / \sqrt{2}\left\{D_{m,-1}^{j *}(\hat{\mathbf{r}}) \mathbf{e}_{-1}(\hat{\mathbf{r}})-D_{m, 1}^{j *}(\hat{\mathbf{r}}) \mathbf{e}_{+1}(\hat{\mathbf{r}})\right\} \\
& \mathbf{Y}_{j m}^{(e)}(\hat{\mathbf{r}})=N_{j} / \sqrt{2}\left\{D_{m,-1}^{j *}(\hat{\mathbf{r}}) \mathbf{e}_{-1}(\hat{\mathbf{r}})+D_{m, 1}^{j *}(\hat{\mathbf{r}}) \mathbf{e}_{+1}(\hat{\mathbf{r}})\right\} \\
& \mathbf{Y}_{j m}^{(0)}(\hat{\mathbf{r}})=N_{j} D_{m, 0}^{j *}(\hat{\mathbf{r}}) \mathbf{e}_{0}(\hat{\mathbf{r}})=Y_{j m}(\hat{\mathbf{r}}) \hat{\mathbf{r}}, \quad N_{j}=[(2 j+1) / 4 \pi]^{1 / 2},
\end{aligned}
$$

where $\mathbf{Y}_{j m}^{(m)}, \mathbf{Y}_{j m}^{(e)}$ and $\mathbf{Y}_{j m}^{(0)}$ are electric, magnetic and longitudinal harmonics, respectively; $\mathbf{e}_{ \pm 1}(\hat{\mathbf{r}})=\mp\left(\mathbf{e}_{x}(\hat{\mathbf{r}}) \pm i \mathbf{e}_{y}(\hat{\mathbf{r}})\right) / \sqrt{2} ; \mathbf{e}_{x}(\hat{\mathbf{r}}) \equiv \hat{\boldsymbol{\vartheta}}=(\cos \theta \cos \phi, \cos \theta \sin \phi,-\sin \theta), \mathbf{e}_{y}(\hat{\mathbf{r}}) \equiv \hat{\boldsymbol{\varphi}}=$ $(-\sin \phi, \cos \phi, 0)$ are the unit vectors tangential to the sphere; $\phi(\theta)$ is the azimuthal (polar) angle of the unit vector $\hat{\mathbf{r}}=\mathbf{r} / r=(\sin \theta \cos \phi, \sin \theta \sin \phi, \cos \theta) \equiv \mathbf{e}_{0}(\hat{\mathbf{r}}) \equiv \mathbf{e}_{z}(\hat{\mathbf{r}}) ; f(\hat{\mathbf{r}}) \equiv f(\phi, \theta)$. (Hats will denote unit vectors and an asterisk will indicate complex conjugation).

Note that, for the irreducible representation of the rotation group with the angular number $j$, the $D$-functions, $D_{m \nu}^{j}(\alpha, \beta, \gamma)=\exp (-i m \alpha) d_{m \mu}^{j}(\beta) \exp (-i \mu \gamma)$, give the elements of the rotation matrix parametrized by the three Euler angles [42,43]: $\alpha, \beta$ and $\gamma$. In formulas (3) and throughout this paper, we assume that $\gamma=0$ and $D_{m \nu}^{j}(\hat{\mathbf{r}}) \equiv D_{m \nu}^{j}(\phi, \theta, 0)$. These $D$-functions meet the following orthogonality relations $[42,43]$

$$
\left\langle D_{m \nu}^{j *}(\hat{\mathbf{r}}) D_{m^{\prime} \nu}^{j^{\prime}}(\hat{\mathbf{r}})\right\rangle_{\hat{\mathbf{r}}}=\frac{4 \pi}{2 j+1} \delta_{j j^{\prime}} \delta_{m m^{\prime}}
$$

where $\langle f\rangle_{\hat{\mathbf{r}}} \equiv \int_{0}^{2 \pi} \mathrm{d} \phi \int_{0}^{\pi} \sin \theta \mathrm{d} \theta f(\hat{\mathbf{r}})$. The orthogonality condition (4) and Eqs. (3) show that a set of vector spherical harmonics is orthonormal:

$$
\left\langle\mathbf{Y}_{j m}^{(\alpha) *}(\hat{\mathbf{r}}) \cdot \mathbf{Y}_{j^{\prime} m^{\prime}}^{(\beta)}(\hat{\mathbf{r}})\right\rangle_{\hat{\mathbf{r}}}=\delta_{\alpha \beta} \delta_{j j^{\prime}} \delta_{m m^{\prime}}
$$

It can be shown [38] that the vector spherical harmonics (3) can also be recast into the well-known standard form [44]:

$$
\begin{aligned}
& \mathbf{Y}_{j m}^{(m)}(\hat{\mathbf{r}})=n_{j} \mathbf{L} Y_{j m}=-i \hat{\mathbf{r}} \times \mathbf{Y}_{j m}^{(e)}, \\
& \mathbf{Y}_{j m}^{(e)}(\hat{\mathbf{r}})=n_{j} r \nabla Y_{j m}=-i \hat{\mathbf{r}} \times \mathbf{Y}_{j m}^{(m)}, \quad n_{j} \equiv[j(j+1)]^{-1 / 2},
\end{aligned}
$$

where $\partial_{x}$ stands for a derivative with respect to $x$ and $Y_{j m}(\hat{\mathbf{r}}) \equiv Y_{j m}(\phi, \theta)$ is the normalized spherical function; $\mathbf{L}$ is the operator of angular momentum given by:

$$
i \mathbf{L}=\mathbf{r} \times \boldsymbol{\nabla}=\hat{\boldsymbol{\varphi}} \partial_{\theta}-\hat{\boldsymbol{\vartheta}}[\sin \theta]^{-1} \partial_{\phi}
$$

The vector wave functions, $\mathbf{M}_{j m}^{(\alpha)}$ and $\mathbf{N}_{j m}^{(\alpha)}$, are the solenoidal solutions of the vector Helmholtz equation that can be derived (a discussion of the procedure can be found, e.g., in Ref. [45]) from solutions of the scalar Helmholtz equation, $\left(\nabla^{2}+k^{2}\right) \psi(\mathbf{r})=0$, taken in the form:

$$
\psi_{j m}^{(\alpha)}=n_{j} z_{j}^{(\alpha)}(k r) \mathrm{Y}_{j m}(\hat{\mathbf{r}}), \quad n_{j} \equiv[j(j+1)]^{-1 / 2},
$$

where $z_{j}^{(\alpha)}(x)$ is either a spherical Bessel function, $j_{j}(x)=[\pi /(2 x)]^{1 / 2} J_{j+1 / 2}(x)$, or a spherical Hankel function [46], $h_{j}^{(1,2)}(x)=[\pi /(2 x)]^{1 / 2} H_{j+1 / 2}^{(1,2)}(x)$. 
In the far field region $(\rho \gg 1)$, the asymptotic behavior of the spherical Bessel and Hankel functions is known [46]:

$$
\begin{aligned}
& i^{j+1} h_{j}^{(1)}(\rho), i^{j} D h_{j}^{(1)}(\rho) \sim \exp (i \rho) / \rho \\
& (-i)^{j+1} h_{j}^{(2)}(\rho),(-i)^{j} D h_{j}^{(2)}(\rho) \sim \exp (-i \rho) / \rho, \\
& i^{j+1} j_{j}(\rho), i^{j+1} D j_{j+1}(\rho) \sim\left[\exp (i \rho)-(-1)^{j} \exp (-i \rho)\right] /(2 \rho) .
\end{aligned}
$$

So, the spherical Hankel functions of the first kind, $h_{j}^{(1)}(\rho)$, describe the outgoing waves, whereas those of the second kind, $h_{j}^{(2)}(\rho)$, represent the incoming waves.

Thus, outside the scatterer, the optical field is the sum of the incident wave field with $z_{j}^{\text {(inc) }}(\rho)=j_{j}(\rho)$ and the scattered waves with $z_{j}^{\text {(sca) }}(\rho)=h_{j}^{(1)}(\rho)$, as required by the Sommerfeld radiation condition. The incident field is the field that would exist without a scatterer and therefore includes both incoming and outgoing parts (see Eq. (12)) because, without scattering, what comes in must go outwards again. As opposed to the spherical Hankel functions that are singular at the origin, the incident wave field should be finite everywhere, and thus, is described by the regular Bessel functions $j_{j}(\rho)$.

Now the incident wave is characterized by amplitudes $\alpha_{j m}^{(\mathrm{inc})}, \beta_{j m}^{(\mathrm{inc})}$ and the scattered outgoing waves are similarly characterized by amplitudes $\alpha_{j m}^{(\mathrm{sca})}, \beta_{j m}^{\text {(sca) }}$. As long as the scattering problem is linear, the coefficients $\alpha_{j m}^{(\mathrm{sca})}$ and $\beta_{j m}^{(\mathrm{sca})}$ can be written as linear combinations of $\alpha_{j m}^{(\mathrm{inc})}$ and $\beta_{j m}^{\text {(inc) }}$ :

$$
\begin{aligned}
& \alpha_{j m}^{\text {(sca) }}=\sum_{j^{\prime}, m^{\prime}}\left[T_{j m, j^{\prime} m^{\prime}}^{11} \alpha_{j^{\prime} m^{\prime}}^{(\mathrm{inc})}+T_{j m, j^{\prime} m^{\prime}}^{12} \beta_{j^{\prime} m^{\prime}}^{(\mathrm{inc})}\right], \\
& \beta_{j m}^{\text {(sca) }}=\sum_{j^{\prime}, m^{\prime}}\left[T_{j m, j^{\prime} m^{\prime}}^{21} \alpha_{j^{\prime} m^{\prime}}^{(\mathrm{inc})}+T_{j m, j^{\prime} m^{\prime}}^{22} \beta_{j^{\prime} m^{\prime}}^{(\mathrm{inc})}\right] .
\end{aligned}
$$

These formulas define the elements of the T-matrix in the most general case.

In general, the scattering process mixes angular momenta [6]. The light scattering from uniformly anisotropic scatterers $[10,11,47,48]$ provides an example of such a scattering process. By contrast, in simpler scattering processes, such angular momentum mixing does not take place. For example, radial anisotropy maintains the spherical symmetry of the scatterer $[8,10,13]$. The $T$-matrix of a spherically symmetric scatterer is diagonal over the angular momenta and the azimuthal numbers: $T_{j j^{\prime}, m m^{\prime}}^{n n^{\prime}}=\delta_{j j^{\prime}} \delta_{m m^{\prime}} T_{j}^{n n^{\prime}}$.

In order to calculate the elements of $T$-matrix and the coefficients $\alpha_{j m}^{(p)}$ and $\beta_{j m}^{(p)}$, we need to use the continuity for the tangential components of the electric and magnetic fields as boundary conditions at $r=R_{p}\left(\rho=k_{\text {med }} R_{p} \equiv x\right)$. Thus, the coefficients of the expansion for the wave field inside the scatterer, $\alpha_{j m}^{(p)}$ and $\alpha_{j m}^{(p)}$, are expressed in terms of the coefficients describing the incident light as follows:

$$
\begin{aligned}
& i \alpha_{j m}^{(p)}=\frac{\alpha_{j m}^{(\mathrm{inc})}}{\mu^{-1} v_{j}(x) u_{j}^{\prime}(n x)-n^{-1} v_{j}^{\prime}(x) u_{j}(n x)}, \quad \mu=\mu_{p} / \mu_{\mathrm{med}}, \\
& i \beta_{j m}^{(p)}=\frac{\beta_{j m}^{(\mathrm{inc})}}{n^{-1} v_{j}(x) u_{j}^{\prime}(n x)-\mu^{-1} v_{j}^{\prime}(x) u_{j}(n x)}, \quad n=n_{p} / n_{\mathrm{med}},
\end{aligned}
$$


where $x=k_{\text {med }} R_{p}, u_{j}(x)=x j_{j}(x)$ and $v_{j}(x)=x h_{j}^{(1)}(x)$. A similar result relates the scattered and incident waves:

$$
\begin{aligned}
& \alpha_{j m}^{\text {(sca) }}=T_{j}^{11} \alpha_{j m}^{(\mathrm{inc})}=\frac{n^{-1} u_{j}^{\prime}(x) u_{j}(n x)-\mu^{-1} u_{j}(x) u_{j}^{\prime}(n x)}{\mu^{-1} v_{j}(x) u_{j}^{\prime}(n x)-n^{-1} v_{j}^{\prime}(x) u_{j}(n x)} \alpha_{j m}^{\text {(inc) }}, \\
& \beta_{j m}^{\text {(sca) }}=T_{j}^{22} \beta_{j m}^{\text {(inc) }}=\frac{\mu^{-1} u_{j}(x) u_{j}^{\prime}(n x)-n^{-1} u_{j}^{\prime}(x) u_{j}(n x)}{n^{-1} v_{j}(x) u_{j}^{\prime}(n x)-\mu^{-1} v_{j}^{\prime}(x) u_{j}(n x)} \beta_{j m}^{\text {(inc) }},
\end{aligned}
$$

defining the $T$-matrix for the simplest case of a spherically symmetric scatterer. In addition, since the parity of electric and magnetic harmonics with respect to the spatial inversion $\hat{\mathbf{r}} \rightarrow-\hat{\mathbf{r}}$ $(\{\phi, \theta\} \rightarrow\{\phi+\pi, \pi-\theta\})$ is different:

$$
\mathbf{Y}_{j m}^{(m)}(-\hat{\mathbf{r}})=(-1)^{j} \mathbf{Y}_{j m}^{(m)}(\hat{\mathbf{r}}), \quad \mathbf{Y}_{j m}^{(e)}(-\hat{\mathbf{r}})=(-1)^{j+1} \mathbf{Y}_{j m}^{(e)}(\hat{\mathbf{r}})
$$

where $f(\hat{\mathbf{r}}) \equiv f(\phi, \theta)$ and $f(-\hat{\mathbf{r}}) \equiv f(\phi+\pi, \pi-\theta)$, they do not mix provided the mirror symmetry has not been broken. In this case the $T$-matrix is diagonal and $T_{j}^{12}=T_{j}^{21}=0$. The diagonal elements $T_{j}^{11} \equiv a_{j}$ and $T_{j}^{22} \equiv b_{j}$ are also called the Mie coefficients.

\section{Far-field matching}

The formulas (14)-(17) are useful only if the expansion for the incident light beam is known. First we briefly review the most studied and fundamentally important case where the incident light is represented by a plane wave.

The electric field of a transverse plane wave propagating along the direction specified by a unit vector $\hat{\mathbf{k}}_{\text {inc }}$ is:

$$
\mathbf{E}_{\mathrm{inc}}=\mathbf{E}^{(\mathrm{inc})} \exp \left(i \mathbf{k}_{\mathrm{inc}} \cdot \mathbf{r}\right), \quad \mathbf{E}^{(\mathrm{inc})}=\sum_{\nu= \pm 1} E_{\nu}^{(\mathrm{inc})} \mathbf{e}_{\nu}\left(\hat{\mathbf{k}}_{\mathrm{inc}}\right), \quad \mathbf{k}_{\mathrm{inc}}=k \hat{\mathbf{k}}_{\mathrm{inc}} .
$$

where the basis vectors $\mathbf{e}_{ \pm 1}\left(\hat{\mathbf{k}}_{\text {inc }}\right)$ are perpendicular to $\hat{\mathbf{k}}_{\text {inc }}$. Then, the vector version of the well-known Rayleigh expansion (see, for example, $[2,10,38]$ ) immediately gives the expansion coefficients for the plane wave:

$$
\alpha_{j m}^{(\mathrm{inc})}=i \alpha_{j} \sum_{\nu= \pm 1} D_{m \nu}^{j}\left(\hat{\mathbf{k}}_{\mathrm{inc}}\right) \nu E_{\nu}^{(\mathrm{inc})}, \quad \beta_{j m}^{(\mathrm{inc})}=-\alpha_{j} \sum_{\nu= \pm 1} D_{m \nu}^{j}\left(\hat{\mathbf{k}}_{\mathrm{inc}}\right) E_{\nu}^{(\mathrm{inc})},
$$

where $\alpha_{j}=i^{j+1}[2 \pi(2 j+1)]^{1 / 2}$.

Now, we consider a more general, case where an incident electromagnetic wave is written as a superposition of propagating plane waves:

$$
\begin{aligned}
& \mathbf{E}_{\text {inc }}(\mathbf{r}) \equiv \mathbf{E}_{\text {inc }}(\rho, \hat{\mathbf{r}})=\left\langle\exp (i \rho \hat{\mathbf{k}} \cdot \hat{\mathbf{r}}) \mathbf{E}_{\mathrm{inc}}(\hat{\mathbf{k}})\right\rangle_{\hat{\mathbf{k}}}, \quad \mathbf{E}_{\mathrm{inc}}(\hat{\mathbf{k}})=\sum_{\nu= \pm 1} E_{\nu}(\hat{\mathbf{k}}) \mathbf{e}_{\nu}(\hat{\mathbf{k}}), \\
& \mathbf{H}_{\mathrm{inc}}(\mathbf{r}) \equiv \mathbf{H}_{\mathrm{inc}}(\rho, \hat{\mathbf{r}})=\frac{n}{\mu}\left\langle\exp (i \rho \hat{\mathbf{k}} \cdot \hat{\mathbf{r}})\left[\hat{\mathbf{k}} \times \mathbf{E}_{\mathrm{inc}}(\hat{\mathbf{k}})\right]\right\rangle_{\hat{\mathbf{k}}}
\end{aligned}
$$

where $\langle f\rangle_{\hat{\mathbf{k}}} \equiv \int_{0}^{2 \pi} \mathrm{d} \phi_{k} \int_{0}^{\pi} \sin \theta_{k} \mathrm{~d} \theta_{k} f$.

Our first step is to examine asymptotic behavior of the wave field (21) in the far-field region, $\rho \gg 1$. The results can be easily obtained by using the asymptotic formula for a plane wave (see, e.g., [4])

$$
\exp (i \rho \hat{\mathbf{k}} \cdot \hat{\mathbf{r}}) \sim \frac{-2 \pi i}{\rho}[\exp (i \rho) \delta(\hat{\mathbf{k}}-\hat{\mathbf{r}})-\exp (-i \rho) \delta(\hat{\mathbf{k}}+\hat{\mathbf{r}})] \quad \text { at } \rho \gg 1
$$


where $\delta(\hat{\mathbf{k}} \mp \hat{\mathbf{r}})$ is the solid angle Dirac $\delta$-function symbolically defined through the expansion:

$$
\delta(\hat{\mathbf{k}} \mp \hat{\mathbf{r}})=\sum_{l=0}^{\infty} \sum_{m=-l}^{l} Y_{l m}( \pm \hat{\mathbf{r}}) Y_{l m}^{*}(\hat{\mathbf{k}}) .
$$

Applying relation (22) to the plane wave superposition (21a) gives the electric field of the incident wave in the far-field region:

$$
\begin{aligned}
& \mathbf{E}_{\text {inc }}(\rho, \hat{\mathbf{r}}) \sim \mathbf{E}_{\text {inc }}^{(\infty)}(\rho, \hat{\mathbf{r}})=\frac{1}{\rho}\left[\exp (i \rho) \mathbf{E}_{\text {out }}^{(\text {inc })}(\hat{\mathbf{r}})+\exp (-i \rho) \mathbf{E}_{\text {in }}^{(\text {inc })}(\hat{\mathbf{r}})\right], \\
& \mathbf{E}_{\text {in }}^{\text {inc })}(\hat{\mathbf{r}})=-\mathbf{E}_{\text {out }}^{(\text {inc) }}(-\hat{\mathbf{r}}),
\end{aligned}
$$

where $\mathbf{E}_{\text {out }}(\hat{\mathbf{r}})$ is the far-field angular distribution for the outgoing part of the electric field of the incident wave:

$$
\mathbf{E}_{\text {out }}^{(\text {inc) }}(\hat{\mathbf{r}})=-2 \pi i \mathbf{E}_{\text {inc }}(\hat{\mathbf{r}})=E_{\theta}^{(\text {out })}(\hat{\mathbf{r}}) \mathbf{e}_{\theta}(\hat{\mathbf{r}})+E_{\phi}^{(\text {out })}(\hat{\mathbf{r}}) \mathbf{e}_{\phi}(\hat{\mathbf{r}}),
$$

whereas the incoming part of the incident wave is described by the far-field angular distribution $\mathbf{E}_{\mathrm{in}}^{(\mathrm{inc})}(\hat{\mathbf{r}})$.

The result for the far-field distribution of the magnetic field (21b) can be written in the similar form:

$$
\begin{aligned}
& \mathbf{H}_{\mathrm{inc}}(\rho, \hat{\mathbf{r}}) \sim \mathbf{H}_{\mathrm{inc}}^{(\infty)}(\rho, \hat{\mathbf{r}})=\frac{1}{\rho}\left[\exp (i \rho) \mathbf{H}_{\text {out }}^{(\mathrm{inc})}(\hat{\mathbf{r}})+\exp (-i \rho) \mathbf{H}_{\mathrm{in}}^{(\mathrm{inc})}(\hat{\mathbf{r}})\right], \\
& \mathbf{H}_{\mathrm{in}}^{(\mathrm{inc})}(\hat{\mathbf{r}})=-\mathbf{H}_{\text {out }}^{(\mathrm{inc})}(-\hat{\mathbf{r}}), \\
& \mu / n \mathbf{H}_{\text {out }}^{(\mathrm{inc})}(\hat{\mathbf{r}})=\hat{\mathbf{r}} \times \mathbf{E}_{\text {out }}^{(\mathrm{inc})}(\hat{\mathbf{r}}), \quad \mu / n \mathbf{H}_{\mathrm{in}}^{(\mathrm{inc})}(\hat{\mathbf{r}})=\hat{\mathbf{r}} \times \mathbf{E}_{\text {out }}^{(\mathrm{inc})}(-\hat{\mathbf{r}}) .
\end{aligned}
$$

Formulas (24)-(29) explicitly show that, in the far-field region, the incident wave field is defined by the angular distribution of the outgoing wave (26).

Alternatively, the far-field distribution of an incident light beam, $\mathbf{E}_{\text {out }}^{(\mathrm{inc})}(\hat{\mathbf{r}})$, can be found from the expansion over the vector spherical harmonics (2a). The far-field asymptotics for the vector wave functions that enter the expansion for the incident wave (2):

$$
\begin{aligned}
& \mathbf{M}_{j m}^{(\mathrm{inc})}(\rho, \hat{\mathbf{r}}) \sim \frac{(-i)^{j+1}}{2 \rho}\left[\exp (i \rho) \mathbf{Y}_{j m}^{(m)}(\hat{\mathbf{r}})-\exp (-i \rho) \mathbf{Y}_{j m}^{(m)}(-\hat{\mathbf{r}})\right], \\
& \mathbf{N}_{j m}^{(\mathrm{inc})}(\rho, \hat{\mathbf{r}}) \sim \frac{(-i)^{j}}{2 \rho}\left[\exp (i \rho) \mathbf{Y}_{j m}^{(e)}(\hat{\mathbf{r}})-\exp (-i \rho) \mathbf{Y}_{j m}^{(e)}(-\hat{\mathbf{r}})\right],
\end{aligned}
$$

can be derived from Eqs. (2c)-(2d) with the help of the far-field relation (12). Substituting Eqs. (30) and (31) into expansion (2a) gives the far-field distribution of the form (24) with:

$$
\mathbf{E}_{\text {out }}^{(\mathrm{inc})}(\hat{\mathbf{r}})=2^{-1} \sum_{j m}(-i)^{j+1}\left[\alpha_{j m}^{(\mathrm{inc})} \mathbf{Y}_{j m}^{(m)}(\hat{\mathbf{r}})+i \beta_{j m}^{(\mathrm{inc})} \mathbf{Y}_{j m}^{(e)}(\hat{\mathbf{r}})\right]
$$

The coefficients of the incident wave can now easily be found as the Fourier coefficients of the far-field angular distribution, $\mathbf{E}_{\text {out }}$, expanded using the vector spherical harmonics basis (3). The final result reads:

$$
\begin{aligned}
& \alpha_{j m}^{(\mathrm{inc})}=2 i^{j+1}\left\langle\mathbf{Y}_{j m}^{(m) *}(\hat{\mathbf{r}}) \cdot \mathbf{E}_{\mathrm{out}}^{(\mathrm{inc})}(\hat{\mathbf{r}})\right\rangle_{\hat{\mathbf{r}}}=i \alpha_{j} \sum_{\nu= \pm 1} \nu\left\langle D_{m \nu}^{j}(\hat{\mathbf{k}}) E_{\nu}(\hat{\mathbf{k}})\right\rangle_{\hat{\mathbf{k}}} \\
& \beta_{j m}^{(\mathrm{inc})}=2 i^{j}\left\langle\mathbf{Y}_{j m}^{(e) *}(\hat{\mathbf{r}}) \cdot \mathbf{E}_{\mathrm{out}}^{(\mathrm{inc})}(\hat{\mathbf{r}})\right\rangle_{\hat{\mathbf{r}}}=-\alpha_{j} \sum_{\nu= \pm 1}\left\langle D_{m \nu}^{j}(\hat{\mathbf{k}}) E_{\nu}(\hat{\mathbf{k}})\right\rangle_{\hat{\mathbf{k}}}
\end{aligned}
$$


A comparison between the expressions on the right hand side of Eq. (33) and those for the plane wave (20) shows that, in agreement with the representation (21a), the result for plane waves represents the limiting case where the angular distribution is singular: $E_{\nu}(\hat{\mathbf{k}})=E_{\nu}^{(\text {inc })} \delta\left(\hat{\mathbf{k}}-\hat{\mathbf{k}}_{\text {inc }}\right)$. form:

By using Eqs. (6) and (7) formulas (33) can conveniently be rewritten in the explicit

$$
\begin{aligned}
& \alpha_{j m}^{(\mathrm{inc})}=2 n_{j} i^{j+1}\left\langle Y_{j m}^{*}(\hat{\mathbf{r}})\left(\mathbf{L} \cdot \mathbf{E}_{\text {out }}^{(\mathrm{inc})}(\hat{\mathbf{r}})\right)\right\rangle_{\hat{\mathbf{r}}}= \\
& 2 n_{j} i^{j} \int_{0}^{2 \pi} \mathrm{d} \phi \int_{0}^{\pi} \mathrm{d} \theta Y_{j m}^{*}(\phi, \theta)\left[\partial_{\theta}\left(\sin \theta E_{\phi}^{(\text {out })}\right)-\partial_{\phi} E_{\theta}^{(\text {out })}\right], \\
& \beta_{j m}^{(\mathrm{inc})}=-2 n_{j} i^{j}\left\langle Y_{j m}^{*}(\hat{\mathbf{r}})\left(r \boldsymbol{\nabla} \cdot \mathbf{E}_{\mathrm{out}}^{(\mathrm{inc})}(\hat{\mathbf{r}})\right)\right\rangle_{\hat{\mathbf{r}}}= \\
& -2 n_{j} i^{j} \int_{0}^{2 \pi} \mathrm{d} \phi \int_{0}^{\pi} \mathrm{d} \theta Y_{j m}^{*}(\phi, \theta)\left[\partial_{\theta}\left(\sin \theta E_{\theta}^{(\text {out })}\right)+\partial_{\phi} E_{\phi}^{(\text {out })}\right],
\end{aligned}
$$

which might be useful for computational purposes.

We conclude this section with the remark concerning the effect of translation:

$$
\left\{\mathbf{E}_{\text {inc }}(\mathbf{r}), \mathbf{H}_{\text {inc }}(\mathbf{r})\right\} \rightarrow\left\{\mathbf{E}_{\text {inc }}\left(\mathbf{r}-\mathbf{r}_{p}\right), \mathbf{H}_{\text {inc }}\left(\mathbf{r}-\mathbf{r}_{p}\right)\right\}
$$

on the far-field angular distribution (26). Note that, under the action of transformation (35), the focal plane is displaced from its initial position by the vector $\mathbf{r}_{p}$. From Eqs. (21) and (26), it follows that, for the far-field distribution (26), translation results in a phase shift:

$$
\mathbf{E}_{\text {out }}^{(\text {inc) }}(\hat{\mathbf{r}}) \rightarrow \mathbf{E}_{\text {out }}^{(\mathrm{inc})}\left(\hat{\mathbf{r}}, \mathbf{r}_{p}\right)=\mathbf{E}_{\text {out }}^{(\mathrm{inc})}(\hat{\mathbf{r}}) \exp \left[-i k\left(\mathbf{r}_{p} \cdot \hat{\mathbf{r}}\right)\right] \text {. }
$$

\subsection{Poynting vector, Maxwell's stress tensor and optical force}

From Eqs. (24)-(29), it is not difficult to obtain the far-field expression for the timeaveraged Poynting vector of the incident wave $\mathbf{S}_{\text {inc }}=c /(8 \pi) \operatorname{Re}\left(\mathbf{E}_{\text {inc }} \times \mathbf{H}_{\text {inc }}^{*}\right)$ :

$$
\begin{aligned}
& \mathbf{S}_{\text {inc }}(\rho, \hat{\mathbf{r}}) \sim \mathbf{S}_{\text {inc }}^{(\infty)}(\rho, \hat{\mathbf{r}})=\rho^{-2}\left\{\mathbf{S}_{\text {in }}^{(\text {inc })}(\hat{\mathbf{r}})+\mathbf{S}_{\text {out }}^{(\text {inc })}(\hat{\mathbf{r}})\right\}, \\
& \mathbf{S}_{\text {in }}^{(\text {inc) }}(\hat{\mathbf{r}})=-\mathbf{S}_{\text {out }}^{(\text {inc) }}(-\hat{\mathbf{r}}), \quad \mu / n \mathbf{S}_{\text {out }}^{\text {(inc) }}(\hat{\mathbf{r}})=c /(8 \pi)\left|\mathbf{E}_{\text {out }}^{(\text {inc })}(\hat{\mathbf{r}})\right|^{2} \hat{\mathbf{r}},
\end{aligned}
$$

where $\left|\mathbf{E}_{\text {out }}^{\text {(inc) }}(\hat{\mathbf{r}})\right|^{2}=\left(\mathbf{E}_{\text {out }}^{\text {(inc) }}(\hat{\mathbf{r}}) \cdot\left[\mathbf{E}_{\text {out }}^{\text {(inc) }}(\hat{\mathbf{r}})\right]^{*}\right)$. From this expression, it immediately follows that the flux of the Poynting vector for the outgoing wave, $\mathbf{S}_{\text {out }}^{(\mathrm{inc})}(\hat{\mathbf{r}})$, through a sphere $S_{f}$ of sufficiently large radius, $R_{f}$, is exactly balanced by the flux of Poynting vector of the incoming wave, $\mathbf{S}_{\text {in }}^{\text {(inc) }}(\hat{\mathbf{r}})$.

For the total optical field, which is a sum of the incident and scattered wavefields, the electric and magnetic fields in the far-field region can also be separated into incoming and the outgoing portions as follows:

$$
\begin{aligned}
& \mathbf{E}_{\text {tot }}=\mathbf{E}_{\text {inc }}+\mathbf{E}_{\text {sca }} \sim \mathbf{E}_{\text {tot }}^{(\infty)}=\frac{1}{\rho}\left[\exp (i \rho) \mathbf{E}_{\text {out }}(\hat{\mathbf{r}})+\exp (-i \rho) \mathbf{E}_{\text {in }}(\hat{\mathbf{r}})\right], \\
& \mathbf{H}_{\text {tot }}=\mathbf{H}_{\text {inc }}+\mathbf{H}_{\text {sca }} \sim \mathbf{H}_{\text {tot }}^{(\infty)}=\frac{1}{\rho}\left[\exp (i \rho) \mathbf{H}_{\text {out }}(\hat{\mathbf{r}})+\exp (-i \rho) \mathbf{H}_{\text {in }}(\hat{\mathbf{r}})\right], \\
& \mu / n \mathbf{H}_{\text {out }}(\hat{\mathbf{r}})=\hat{\mathbf{r}} \times \mathbf{E}_{\text {out }}(\hat{\mathbf{r}}), \quad \mu / n \mathbf{H}_{\text {in }}(\hat{\mathbf{r}})=-\hat{\mathbf{r}} \times \mathbf{E}_{\text {in }}(\hat{\mathbf{r}}), \\
& \mathbf{E}_{\text {out }}(\hat{\mathbf{r}})=\mathbf{E}_{\text {out }}^{(\text {inc })}(\hat{\mathbf{r}})+\mathbf{E}_{\text {out }}^{(\text {sca })}(\hat{\mathbf{r}}), \quad \mathbf{E}_{\text {in }}(\hat{\mathbf{r}})=-\mathbf{E}_{\text {out }}^{(\text {inc })}(-\hat{\mathbf{r}}),
\end{aligned}
$$


where, similar to the case of the incident wave (32), the far-field angular distribution of the scattered wave, $\mathbf{E}_{\text {out }}^{(\mathrm{sca})}(\hat{\mathbf{r}})$, is determined by the expansion in vector spherical harmonics:

$$
\mathbf{E}_{\text {out }}^{(\mathrm{sca})}(\hat{\mathbf{r}})=\sum_{j m}(-i)^{j+1}\left[\alpha_{j m}^{(\mathrm{sca})} \mathbf{Y}_{j m}^{(m)}(\hat{\mathbf{r}})+i \beta_{j m}^{(\mathrm{sca})} \mathbf{Y}_{j m}^{(e)}(\hat{\mathbf{r}})\right]
$$

We can now generalize the Poynting vector expression (37) to the case of the total wavefield given in Eqs. (39)-(41):

$$
\mu / n \mathbf{S}_{\text {tot }}^{(\infty)}(\rho, \hat{\mathbf{r}})=\frac{c}{8 \pi \rho^{2}}\left\{\left|\mathbf{E}_{\text {out }}(\hat{\mathbf{r}})\right|^{2}-\left|\mathbf{E}_{\text {in }}(\hat{\mathbf{r}})\right|^{2}\right\} \hat{\mathbf{r}}
$$

and use the relations (42) to evaluate the flux of the Poynting vector (44) through the far-field sphere $S_{f}$ of the radius $R_{f}$. The result can be written in the following well-known form:

$$
\begin{aligned}
& \oiint_{S_{f}}\left(\mathbf{S}_{\mathrm{tot}}^{(\infty)} \cdot \mathrm{d} \mathbf{s}\right)=R_{f}^{2}\left\langle\left(\mathbf{S}_{\mathrm{tot}}^{(\infty)}\left(k R_{f}, \hat{\mathbf{r}}\right) \cdot \hat{\mathbf{r}}\right)\right\rangle_{\hat{\mathbf{r}}} \equiv-W_{\mathrm{abs}}=W_{\mathrm{sca}}-W_{\mathrm{ext}}, \\
& W_{\mathrm{sca}}=\frac{c n}{8 \pi \mu k^{2}}\left\langle\left|\mathbf{E}_{\mathrm{out}}^{(\mathrm{sca})}(\hat{\mathbf{r}})\right|^{2}\right\rangle_{\hat{\mathbf{r}}}, \quad W_{\mathrm{ext}}=-\frac{c n}{4 \pi \mu k^{2}} \operatorname{Re}\left\langle\left(\mathbf{E}_{\mathrm{out}}^{(\mathrm{sca})}(\hat{\mathbf{r}}) \cdot\left[\mathbf{E}_{\mathrm{out}}^{(\mathrm{inc})}(\hat{\mathbf{r}})\right]^{*}\right)\right\rangle_{\hat{\mathbf{r}}},
\end{aligned}
$$

where $W_{\text {sca }}$ is the energy scattering rate (the rate at which the scattered energy crosses the sphere in an outward direction), $W_{\text {abs }}$ is the energy absorption rate and $W_{\text {ext }}=W_{\text {sca }}+W_{\text {abs }}$ is the extinction rate. When the scatterer and the surrounding medium are both non-absorbing, the energy absorption rate vanishes, $W_{\mathrm{abs}}=0$, and Eq. (45) yields unitarity relations for the $T$-matrix [4]. In our spherically symmetric case, these are: $\left|2 T_{j}^{11}+1\right|=\left|2 T_{j}^{22}+1\right|=1$.

The far-field angular distributions, $\mathbf{E}_{\text {out }}^{(\mathrm{sca})}(\hat{\mathbf{r}})$ and $\mathbf{E}_{\text {out }}^{(\mathrm{inc})}(\hat{\mathbf{r}})$, also determine the timeaveraged optical force, $\mathbf{F}$, acting upon the particle. This force can be expressed in terms of the time-average of Maxwell's stress tensor $\mathbf{T}_{M}$ :

$$
\mathbf{T}_{M}=\frac{1}{8 \pi} \operatorname{Re}\left\{\epsilon \mathbf{E} \otimes \mathbf{E}^{*}+\mu \mathbf{H} \otimes \mathbf{H}^{*}-\mathbf{I}_{3}\left(\epsilon|\mathbf{E}|^{2}+\mu|\mathbf{H}|^{2}\right) / 2\right\},
$$

where $\mathbf{I}_{3}$ is the $3 \times 3$ identity matrix, as follows:

$$
\mathbf{F}=\oiint_{S_{f}}\left(\mathbf{T}_{M}^{(\infty)} \cdot \mathrm{d} \mathbf{s}\right)
$$

where $\mathbf{T}_{M}^{(\infty)}$ is the Maxwell stress tensor (47) in the far-field region. Substituting Eqs. (39)-(41) into the stress tensor (47) gives the following expression for the dot product:

$$
\left(\mathbf{T}_{M}^{(\infty)} \cdot \hat{\mathbf{r}}\right)=-\frac{\epsilon}{8 \pi \rho^{2}}\left\{\left|\mathbf{E}_{\text {out }}(\hat{\mathbf{r}})\right|^{2}+\left|\mathbf{E}_{\mathrm{in}}(\hat{\mathbf{r}})\right|^{2}\right\} \hat{\mathbf{r}}
$$

that enter the integrand on the right-hand side of Eq. (48). The final result for the optical force reads:

$$
\mathbf{F}\left(\mathbf{r}_{p}\right)=-\frac{\epsilon}{8 \pi k^{2}}\left\{\left\langle\hat{\mathbf{r}}\left|\mathbf{E}_{\text {out }}^{(\mathrm{sca})}\left(\hat{\mathbf{r}}, \mathbf{r}_{p}\right)\right|^{2}\right\rangle_{\hat{\mathbf{r}}}+2\left\langle\hat{\mathbf{r}} \operatorname{Re}\left(\mathbf{E}_{\text {out }}^{(\mathrm{sca})}\left(\hat{\mathbf{r}}, \mathbf{r}_{p}\right) \cdot\left[\mathbf{E}_{\text {out }}^{(\mathrm{inc})}\left(\hat{\mathbf{r}}, \mathbf{r}_{p}\right)\right]^{*}\right)\right\rangle_{\hat{\mathbf{r}}}\right\},
$$

where we have indicated that the net force exerted on the particle depends on the displacement vector $\mathbf{r}_{p}$ describing position of the scatterer with respect to the focal plane. 


\subsection{Remodelled Laguerre-Gaussian beams}

In the paraxial approximation, the beams are described in terms of scalar fields of the form: $u(\mathbf{r}) \exp (i k z)$, where $u(\mathbf{r})$ is a solution of the paraxial Helmholtz equation:

$$
\left[\boldsymbol{\nabla}_{\perp}^{2}+2 i k \partial_{z}\right] u=0, \quad \nabla_{\perp}^{2}=\partial_{x}^{2}+\partial_{y}^{2} .
$$

For LG beams, the solution can be conveniently written in the cylindrical coordinate system, $\left(r_{\perp}, \phi, z\right)$, as follows

$$
\begin{aligned}
& u_{n m}\left(r_{\perp}, \phi, z\right)=|\sigma|^{-1} \psi_{n m}\left(\sqrt{2} r_{\perp} / w\right) \exp \left\{-r_{\perp}^{2} /\left(w_{0}^{2} \sigma\right)+i m \phi-i \gamma_{n m}\right\}, \\
& \sigma \equiv \sigma(z)=1+i z / z_{R}, \quad w \equiv w(z)=w_{0}|\sigma|, \\
& \gamma_{n m} \equiv \gamma_{n m}(z)=(2 n+m+1) \arctan \left(z / z_{R}\right), \quad \psi_{n m}(x)=x^{|m|} L_{n}^{|m|}\left(x^{2}\right),
\end{aligned}
$$

where $L_{n}^{m}$ is the generalized Laguerre polynomial given by [49]:

$$
L_{n}^{m}(x)=(n !)^{-1} x^{-m} \exp (x) \partial_{x}^{n}\left[x^{n+m} \exp (-x)\right],
$$

where $n(m)$ is the radial (azimuthal) mode number; $w_{0}$ is the initial transverse Gaussian half-width (the beam diameter at waist) $z_{R}=k w_{0}^{2} / 2=\left[2 k f^{2}\right]^{-1}$ is the Rayleigh range and $f=\left[k w_{0}\right]^{-1}$ is the focusing parameter.

The problem studied in Refs. [27,29,30] deals with the exact propagation of the optical field in the half-space, $z>0$, when its transverse components at the initial (source) plane, $z=0$, are known. In Ref. [29], the results describing asymptotic behavior of the linearly polarized field:

$$
\mathbf{E}\left(r_{\perp}, \phi, 0\right)=u_{n m}\left(r_{\perp}, \phi, 0\right) \hat{\mathbf{x}}=\psi_{n m}\left(\sqrt{2} r_{\perp} / w_{0}\right) \exp \left\{-r_{\perp}^{2} / w_{0}^{2}+i m \phi\right\} \hat{\mathbf{x}},
$$

were derived using the angular spectrum representation (Debye integrals) and comply with both results of rigorous mathematical analysis performed in Ref. [50] and those obtained using the vectorial Rayleigh-Sommerfeld integrals [27,30]. The resulting expression for the far-field angular distribution can be written in the following form:

$$
\begin{aligned}
& \mathbf{E}_{\text {out }}^{(\mathrm{LG})}(\phi, \theta)=E_{n m}\left(f^{-1} \sin \theta / \sqrt{2}\right) \exp (i m \phi) \mathbf{e}_{\text {out }}, \\
& \mathbf{e}_{\text {out }}=\cos \phi \mathbf{e}_{\theta}(\hat{\mathbf{r}})-\cos \theta \sin \phi \mathbf{e}_{\phi}(\hat{\mathbf{r}})=\cos \theta \hat{\mathbf{x}}-\sin \theta \cos \phi \hat{\mathbf{z}}, \\
& E_{n m}(x)=\frac{x^{m}}{i^{2 n+m+1} 2 f^{2}} L_{n}^{m}\left(x^{2}\right) \exp \left(-x^{2} / 2\right) .
\end{aligned}
$$

We can now combine relations (26) and (21) with the outgoing part of the far-field distribution (55a) to deduce the expression for the electric field of the remodelled LG beam:

$$
\begin{aligned}
& \mathbf{E}_{\mathrm{inc}}^{(\mathrm{LG})}\left(\rho_{\perp}, \phi, \rho_{z}\right)=E_{x}^{(\mathrm{LG})}\left(\rho_{\perp}, \phi, \rho_{z}\right) \hat{\mathbf{x}}+E_{z}^{(\mathrm{LG})}\left(\rho_{\perp}, \phi, \rho_{z}\right) \hat{\mathbf{z}}= \\
& \frac{i}{2 \pi}\left\langle\exp \left[i\left(\rho_{\perp} \sin \theta_{k} \cos \left(\phi-\phi_{k}\right)+\rho_{z} \cos \theta_{k}\right)\right] \mathbf{E}_{\text {out }}^{(\mathrm{LG})}(\hat{\mathbf{k}})\right\rangle_{\hat{\mathbf{k}}}
\end{aligned}
$$

where $\rho_{\perp}=k r_{\perp}$ and $\rho_{z}=k z$.

\subsection{Laser beam symmetries}

In Sec. 3.1, we have shown that the scattering characteristics such as the cross-sections and the radiation force can be expressed in terms of the far-field angular distributions that can be regarded as vector fields on a sphere. Under the action of the orthogonal transformation $M$ : $\hat{\mathbf{r}} \mapsto \hat{\mathbf{r}}^{\prime}=M \hat{\mathbf{r}}$, such fields transform as follows:

$$
\mathbf{E}_{\text {out }}(\hat{\mathbf{r}}) \mapsto \mathbf{E}_{\text {out }}^{\prime}=M \mathbf{E}_{\text {out }}\left(M^{-1} \hat{\mathbf{r}}\right)
$$


From Eqs. (21a) and (50), we derive the following relations:

$$
\mathbf{E}_{\text {inc }}(\mathbf{r}) \mapsto \mathbf{E}_{\text {inc }}^{\prime}=M \mathbf{E}_{\text {inc }}\left(M^{-1} \mathbf{r}\right), \quad \mathbf{F}\left[\mathbf{E}_{\text {out }}\right] \mapsto \mathbf{F}\left[\mathbf{E}_{\text {out }}^{\prime}\right]=M \mathbf{F}\left[\mathbf{E}_{\text {out }}\right],
$$

which define transformations of the incident wave and the optical force.

The symmetry transformation $M_{s}$ for the far-field angular distribution of the incident wave may generally be defined through the symmetry relation:

$$
M_{s} \mathbf{E}_{\text {out }}^{(\mathrm{inc})}\left(M_{s}^{-1} \hat{\mathbf{r}}\right)=U_{s} \mathbf{E}_{\text {out }}^{(\mathrm{inc})}(\hat{\mathbf{r}}),
$$

where $U_{s}$ is the matrix of a unitary transformation. At $\left|\mathbf{r}_{p}\right| \neq 0$, we can use Eq. (36) combined with the orthogonality relation: $\left(\hat{\mathbf{r}} \cdot \mathbf{r}_{p}\right)=\left(M_{s}^{-1} \hat{\mathbf{r}} \cdot M_{s}^{-1} \mathbf{r}_{p}\right)$ to recast the symmetry condition (59) in the form:

$$
U_{s} \mathbf{E}_{\text {out }}^{\text {(inc) }}\left(\hat{\mathbf{r}}, \mathbf{r}_{p}\right)=M_{s} \mathbf{E}_{\text {out }}^{(\text {inc) }}\left(M_{s}^{-1} \hat{\mathbf{r}}, M_{s}^{-1} \mathbf{r}_{p}\right) .
$$

As a direct consequence of the generalized symmetry relation (60) for the optical force, we have:

$$
\mathbf{F}\left(\mathbf{r}_{p}\right)=M_{s} \mathbf{F}\left(M_{s}^{-1} \mathbf{r}_{p}\right), \quad \mathbf{K}\left(\mathbf{r}_{p}\right)=M_{s} \mathbf{K}\left(M_{s}^{-1} \mathbf{r}_{p}\right) M_{s}^{-1},
$$

where the elements of the stiffness (force) matrix $\mathbf{K}\left(\mathbf{r}_{p}\right)$ are given by:

$$
K_{i j}\left(\mathbf{r}_{p}\right)=\partial_{j} F_{i}\left(\mathbf{r}_{p}\right) \text {. }
$$

At equilibria, the force vanishes $\left(\mathbf{F}\left(\mathbf{r}_{\mathrm{eq}}\right)=\mathbf{0}\right)$ and the stiffness matrix, $\mathbf{K}_{\mathrm{eq}}=\mathbf{K}\left(\mathbf{r}_{\mathrm{eq}}\right)$, is known to govern the regime of linearized particle dynamics [51].

For the LG beams with the angular distribution (55a), it can easily be verified if the direction of propagation (the $z$ axis) is the axis of twofold rotational symmetry $C_{2}$ with $C_{2}$ : $\phi \mapsto \phi+\pi$ and $C_{2}=\operatorname{diag}(-1,-1,1)$. From Eq. (55a), we have:

$$
C_{2} \mathbf{E}_{\text {out }}^{(\mathrm{LG})}\left(C_{2} \hat{\mathbf{r}}\right)=C_{2} \mathbf{E}_{\text {out }}^{(\mathrm{LG})}(\phi+\pi, \theta)=(-1)^{m+1} \mathbf{E}_{\text {out }}^{(\mathrm{LG})}(\hat{\mathbf{r}})
$$

When $\mathbf{r}_{d} \| \hat{\mathbf{z}}$ and $C_{2} \mathbf{r}_{d}=\mathbf{r}_{d}$, equation (61) for the twofold symmetry implies that the optical force is directed along the symmetry axis, $\mathbf{F} \| \hat{\mathbf{z}}$, and the stiffness matrix is of the form:

$$
\mathbf{K}=\left(\begin{array}{ccc}
K_{x x} & K_{x y} & 0 \\
K_{y x} & K_{y y} & 0 \\
0 & 0 & K_{z z}
\end{array}\right)
$$

Since $C_{2} \mathbf{Y}_{j m}^{(e, m)}\left(C_{2} \hat{\mathbf{r}}\right)=(-1)^{m} \mathbf{Y}_{j m}^{(e, m)}(\hat{\mathbf{r}})$, for $C_{2}$ symmetric LG beams, the azimuthal numbers of nonvanishing beam shape coefficients are of the same parity (all $m$ are either odd or even).

\section{Near-field nanostructures}

In this section, we present the results of numerical computations for the light scattering problem for the case where the incident wave is represented by the remodelled LG beams (56) with the vanishing radial mode number $n=0$ and the nonzero azimuthal number, $m=m_{\mathrm{LG}} \geq$ 0 . Such beams are also known as purely azimuthal LG beams [52].

In agreement with our symmetry analysis, substituting the far-field distribution (55) into Eq. (34) gives the beam shape coefficients of these beams in the following form:

$$
\begin{aligned}
& \alpha_{j m}^{(\mathrm{inc})}=\alpha_{j, m_{\mathrm{LG}}}^{(+)} \delta_{m, m_{\mathrm{LG}}+1}+\alpha_{j, m_{\mathrm{LG}}^{(-)}} \delta_{m, m_{\mathrm{LG}}-1}, \\
& \beta_{j m}^{(\mathrm{inc})}=\beta_{j, m_{\mathrm{LG}}^{(+)}} \delta_{m, m_{\mathrm{LG}}+1}+\beta_{j, m_{\mathrm{LG}}}^{(-)} \delta_{m, m_{\mathrm{LG}}-1} .
\end{aligned}
$$

Then, the coefficients of expansions (2) describing scattered wave and electromagnetic field inside the scatterer can be evaluated from formulas (14)-(17). 


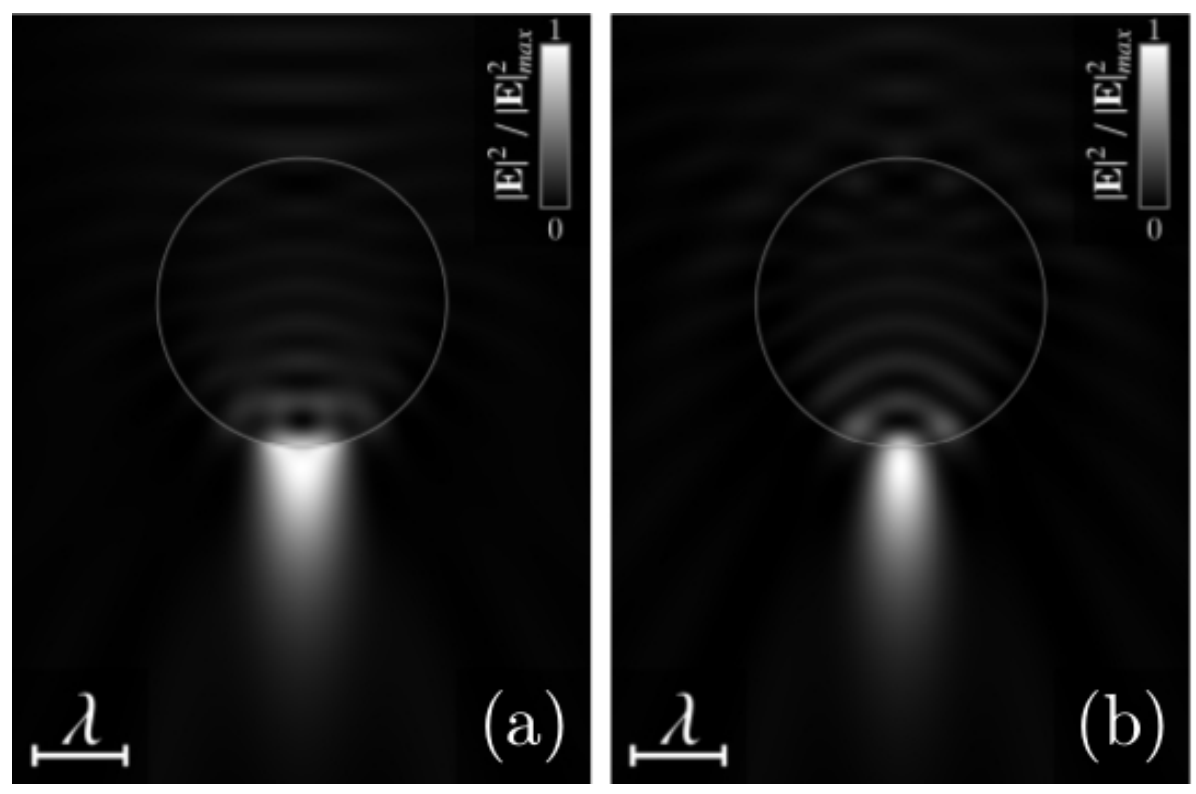

FIG. 1. Near-field intensity distributions of the total wavefield in (a) the $x-z$ plane and (b) the $y-z$ plane for the LG beam with $m_{\mathrm{LG}}=0, f=0.05$ and $\left|\mathbf{r}_{p}\right|=0$. The parameters are: $R_{p}=1.5 \lambda$ is the scatterer radius and $n_{p}=1.3$ $\left(n_{\text {med }}=1.0\right)$ is the refractive index inside (outside) the particle.

\subsection{Photonic nanojets}

For spherical particles illuminated by plane waves, the formation of photonic nanojets and their structure was previously discussed in Refs. [53-55]. Plane waves can be regarded as Gaussian beams with $n=m_{\mathrm{LG}}=0$ and sufficiently small focusing parameter, $f \ll 1$, which is defined after Eq. (53) through the ratio of wavelength, $\lambda$, and the beam diameter at waist, $w_{0}$, $f=(2 \pi)^{-1} \lambda / w_{0}$. This limiting case is illustrated in Fig. 1 which shows the near-field intensity distributions for the total light wavefield in both the $x-z$ and the $y-z$ planes computed at $m_{\mathrm{LG}}=0$ and $f=0.05$ for the spherical particle of the radius $R_{p}=1.5 \lambda$ with the refractive index $n_{p}=1.33$ (water) located in air $\left(n_{\mathrm{m}}=1\right)$.

It can be seen that the distributions are characterized by the presence of elongated focusing zones formed near the shadow surface of the scatterer. The transverse sizes of these zones are smaller than the wavelength of incident light, whereas their longitudinal size in the direction of incidence, which is along the $z$ axis from top to bottom, is relatively large. Such a jetlike light structure is typical for the photonic nanojets. The characteristic length and width of nanojets along with the peak intensity are known to strongly depend on a number of factors, such as the scatterer size $R_{p}$, the particle absorption coefficient and the optical contrast ratio $n_{p} / n_{\mathrm{m}}$. For microspheres, the results of a comprehensive numerical analysis including the case of shell particles are summarized in a recent paper [55].

The effects of non-plane incident waves, such as laser beams on the structure of photonic nanojets, are much less studied. Some theoretical results for tightly focused Gaussian beams are reported in Ref. [56] and the case of Bessel-Gauss beams was studied experimentally in [57].

For the LG beams, we begin with the effects of the azimuthal mode number and describe what happens to the near-field structure shown in Fig. 1 when the azimuthal number takes the smallest nonzero value, $m_{\mathrm{LG}}=1$. The latter represents the simplest case of an optical vortex beam in which, owing to the presence of phase singularity, the intensity of incident light at the beam axis (the $z$ axis) vanishes (see Fig. 2(a)). From Fig. 2, it can be seen that, even though 

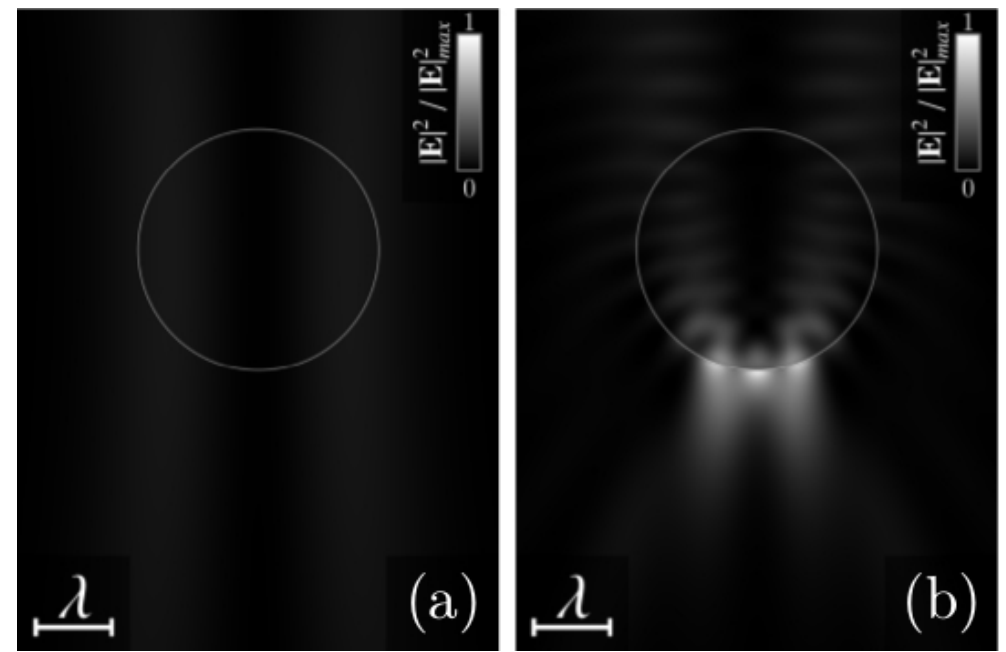

FIG. 2. Near-field intensity distribution in the $x-z$ plane of (a) the incident wave beam and (b) the total wavefield for the LG beam with $m_{\mathrm{LG}}=1, f=0.1$ and $\left|\mathbf{r}_{p}\right|=0$. Other parameters are described in the caption of Fig. 1.

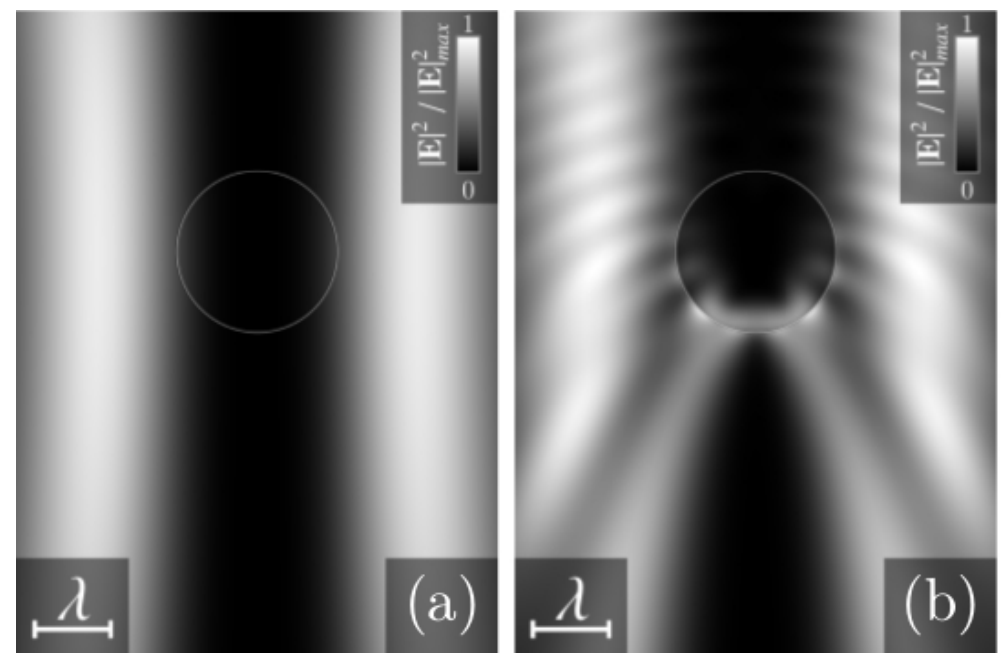

FIG. 3. Near-field intensity distribution in the $x-z$ plane of (a) the incident wave beam and (b) the total wave field for the LG beam with $m_{\mathrm{LG}}=2, f=0.08$ and $\left|\mathbf{r}_{p}\right|=0$.

the bulk part of the scatterer is in the low intensity region surrounding the optical vortex, the scattering process is efficient enough to produce scattered waves that result in the formation of a pronounced jetlike photonic flux, emerging from the surface of the particle's shadow (see Fig. 2(b)).

A comparison between Fig. 2(b) and Fig. 1(a) shows that the three-peak structure of the photonic jet formed with Mie scattering of the optical vortex $\mathrm{LG}$ beam with $m_{\mathrm{LG}}=1$ significantly differs from the well-known shape of the nanojet at $m_{\mathrm{LG}}=0$. Interestingly, similar to the case of Gaussian beams with $m_{\mathrm{LG}}=0$, the focusing zones at $m_{\mathrm{LG}}=1$ involve the beam axis where one of the light intensity peaks is located.

From Fig. 2, it can be seen that, even though the bulk part of the scatterer is in the low intensity region surrounding the optical vortex, the scattering process is efficient enough to produce scattered waves that result in the formation of a pronounced jetlike photonic flux 
emerging from the surface of the particle's shadow (see Fig. 2(b)). Figure 3 demonstrates that, for the weakly focused LG beam with $f=0.08$, this effect can be even more pronounced at $m_{\mathrm{LG}}=2$.

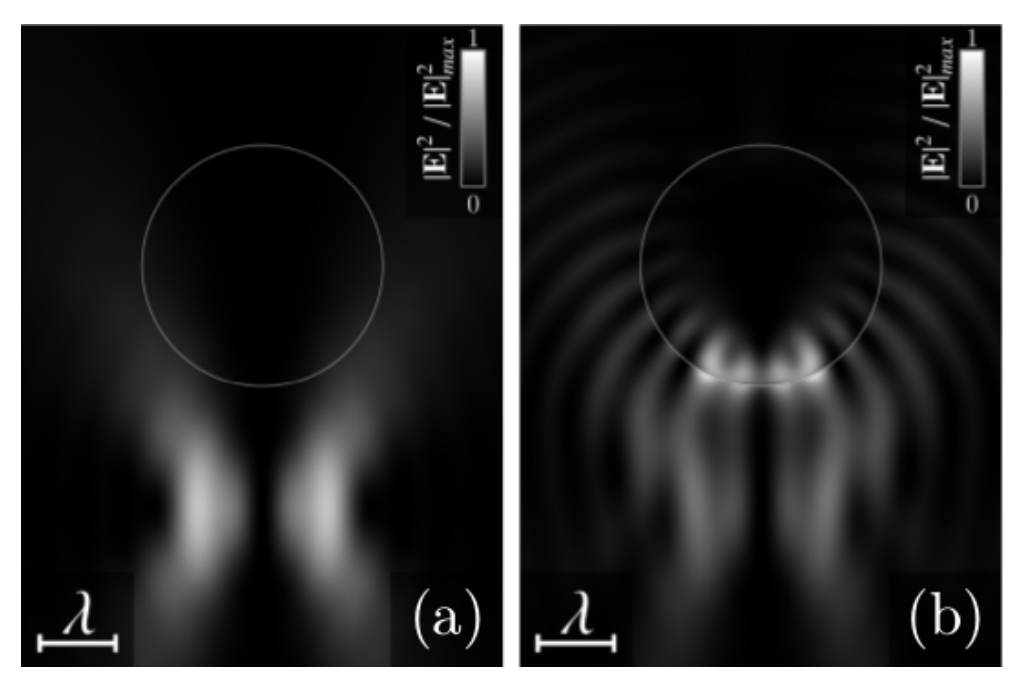

FIG. 4. Near-field intensity distribution in the $x-z$ plane of (a) the incident wave beam and (b) the total wave field for the LG beam with $m_{\mathrm{LG}}=2, f=0.25$ and $\mathbf{r}_{p}=(0,0,3 \lambda)$.

The results for tightly focused LG beams with $m_{\mathrm{LG}}=2$ and $f=0.25$ are shown in Fig. 4. When the displacement vector, $\mathbf{r}_{p}$ defined in Eqs. (35) vanishes, the focal (waist) plane of the incident LG beam is $z=0$ and the bulk part of the four-peak structure of the focusing zones is localized inside of the particles. For $\mathbf{r}_{p}=(0,0,3 \lambda)$, the focal plane, $z=3 \lambda$, is located behind the particle (see Fig. 4(a)). From Fig. 4(b), it is seen that four peaks of light intensity now develop in the immediate vicinity of the scatterer surface.

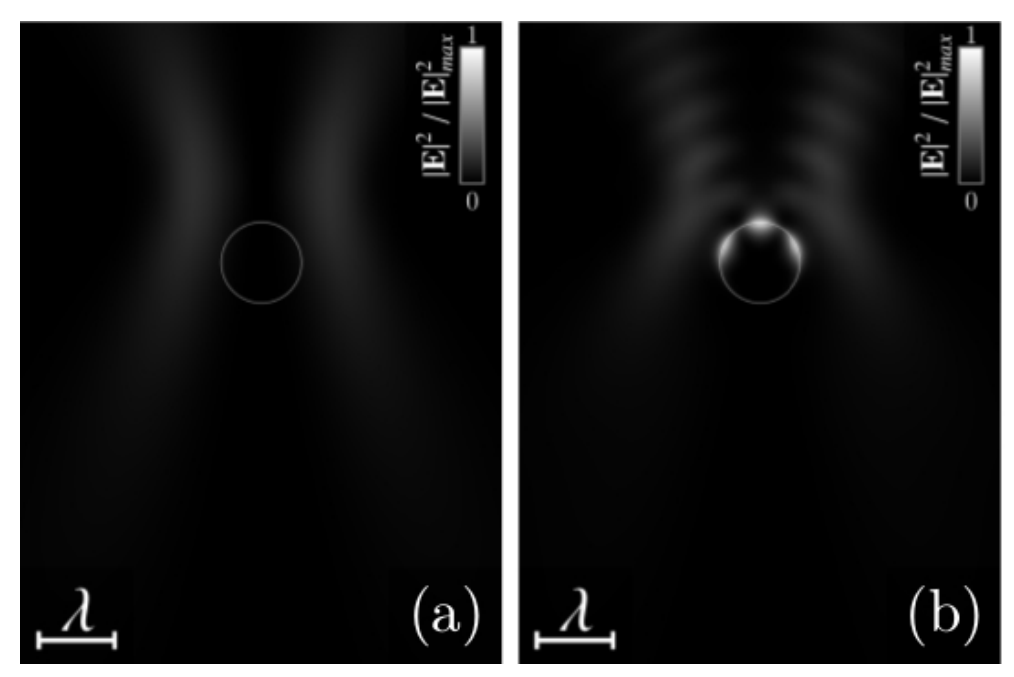

FIG. 5. Near-field intensity distribution in the $x-z$ plane of (a) the incident wave beam and (b) the total wave field for the LG beam with $m_{\mathrm{LG}}=2, f=0.2$ and $\mathbf{r}_{p}=(0,0, \lambda)$. The small scatterer $\left(R_{p}=0.5 \lambda\right)$ is made of the negative index (left-handed) metamaterial with $\epsilon_{p}=\mu_{p}=-1$.

What all the wavefields depicted in Figs. 2(b)-4(b) have in common is that, in contrast to the incident optical vortex beams with $m_{\mathrm{LG}}=1$ and $m_{\mathrm{LG}}=2$, the light intensity at the 
incident beam axis (the $z$ axis) clearly differs from zero. In other words, in the near-field region, the optical vortex with $0<\left|m_{\mathrm{LG}}\right| \leq 2$ has been destroyed by Mie scattering. As it will be explained in the subsequent section this is no longer the case at $m_{\mathrm{LG}} \geq 3$.

In the conclusion of this section, we briefly discuss the structure of the nanojets for scatterers made of the negative index (left-handed or double negative) metamaterial (such materials are reviewed in a number of books and papers [58-61]). This is case where $\operatorname{Re} \epsilon_{p}$ and Re $\mu_{p}$ are both negative. For the limiting case of a nonabsorbing Veselago medium [62] with $\epsilon_{p}=\mu_{p}=-1$, Fig. 5 shows a rather unusual nanojet structure formed in the illuminated part of the small particle as opposed to the structures shown in Figs. 1-4. An important point is that, in real metamaterials, the effects of absorption cannot be neglected. In particular, these effects may prevent formation of jetlike structures near metallic particles and this is why, to the best of our knowledge, the current literature on photonic nanojets has been focused exclusively on the case of dielectric scattering. Figure 6 demonstrates that a jetlike structure may form near the shadow surface of a metallic particle illuminated by a vortex laser beam (the LG beam with $m_{\mathrm{LG}}=1$ ). A comprehensive study of absorption effects in metallic and metamaterial scatterers is well beyond the scope of this paper and the corresponding results will be published elsewhere.

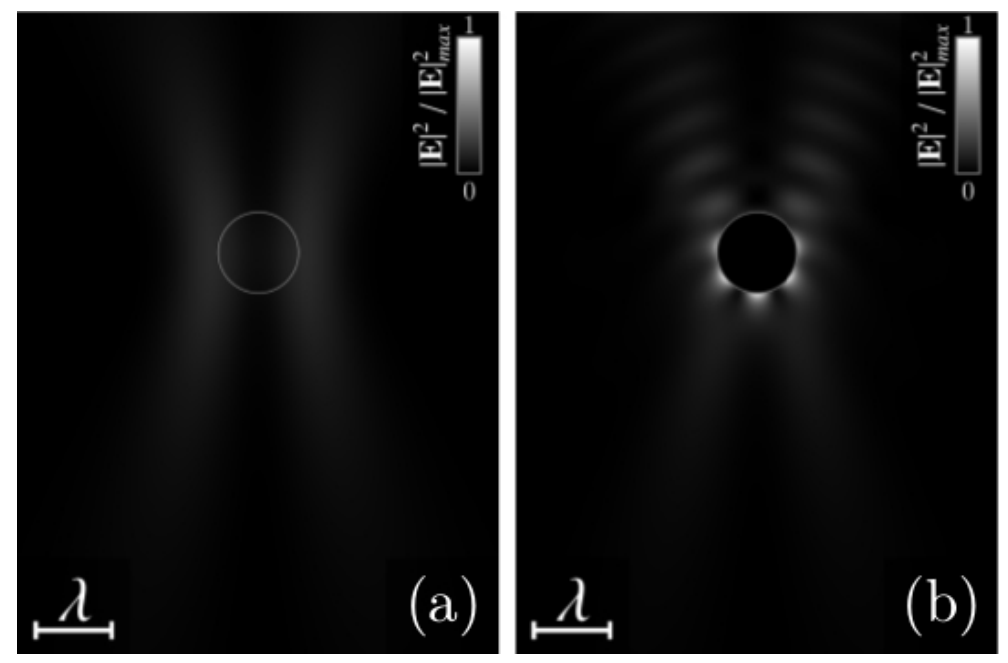

FIG. 6. Near-field intensity distribution in the $x-z$ plane of (a) the incident wave beam and (b) the total wave field for the LG beam with $m_{\mathrm{LG}}=1, f=0.2$ and $\mathbf{r}_{p}=(0,0, \lambda)$. The metallic scatterer $\left(R_{p}=0.5 \lambda\right)$ is made of gold with $\epsilon_{p} \approx-22+1.8 i(\lambda \approx 800 \mathrm{~nm})$.

\subsection{Optical vortices}

In this section, we consider optical vortices and their near-field structure. The optical vortices are known to represent phase singularities of complex-valued scalar waves which are zeros of the wavefield $\psi=|\psi| \exp (i \chi)$ where its phase $\chi$ is undefined. A phase singularity is characterized by the topological vortex charge $m_{V}$, defined as the closed loop contour integral of the wave phase $\chi$ modulo $2 \pi$ :

$$
m_{V}=\frac{1}{2 \pi} \oint_{L} \mathrm{~d} \chi,
$$

where $L$ is the closed path around the singularity.

Optical vortices associated with the individual components of electric field will be of our primary concern. More specifically, we shall examine the optical vortex structure of the 
components $E_{z}$ and $E_{x}$ in the planes $z=z_{0}$ parallel to the $x-y$ plane. Since, in such planes, circles naturally play the role of closed loops, the starting point of our analysis is the electric field vector, expressed as a function of the azimuthal angle $\phi$ in the following form:

$$
\begin{aligned}
& \mathbf{E}=\sum_{\mu=-2}^{2} \mathbf{E}_{\mu} \exp \left[i\left(m_{\mathrm{LG}}+\mu\right) \phi\right], \\
& \mathbf{E}_{ \pm 2}\left\|\hat{\mathbf{x}} \mp i \hat{\mathbf{y}}, \quad \mathbf{E}_{ \pm 1}\right\| \hat{\mathbf{z}}, \quad \mathbf{E}_{0} \perp \hat{\mathbf{z}} .
\end{aligned}
$$

This formula gives the $\phi$ dependence of electric field expansion (2a), in which the coefficients are of the form given by Eq. (65). An immediate consequence of Eq. (67) is that $\mathbf{E}_{\mu}$ can be different from zero on the $z$ axis, $\mathbf{E}_{\mu}(0,0, z) \neq 0$, only if $m_{\mathrm{LG}}+\mu=0$.

From Eq. (68), at $\left|m_{\mathrm{LG}}\right|=1$, the electric field non-vanishing at the beam axis is linearly polarized along the $z$ axis, whereas it is circular polarized at $\left|m_{\mathrm{LG}}\right|=2$. The intensity distributions shown in Figs 1-4 clearly indicate that the $z$ axis is not entirely in the dark region provided that $0 \leq m_{\mathrm{LG}}<3$.

At $\left|m_{\mathrm{LG}}\right| \geq 3$ and $|\mu| \leq 2$, a sum $m_{\mathrm{LG}}+\mu$ cannot be equal to zero and the beam axis is always a nodal line for the components of electric field. For two-dimensional (2D) electric field distributions in planes normal to the $z$ axis, it implies that there is an optical vortex located at the origin.
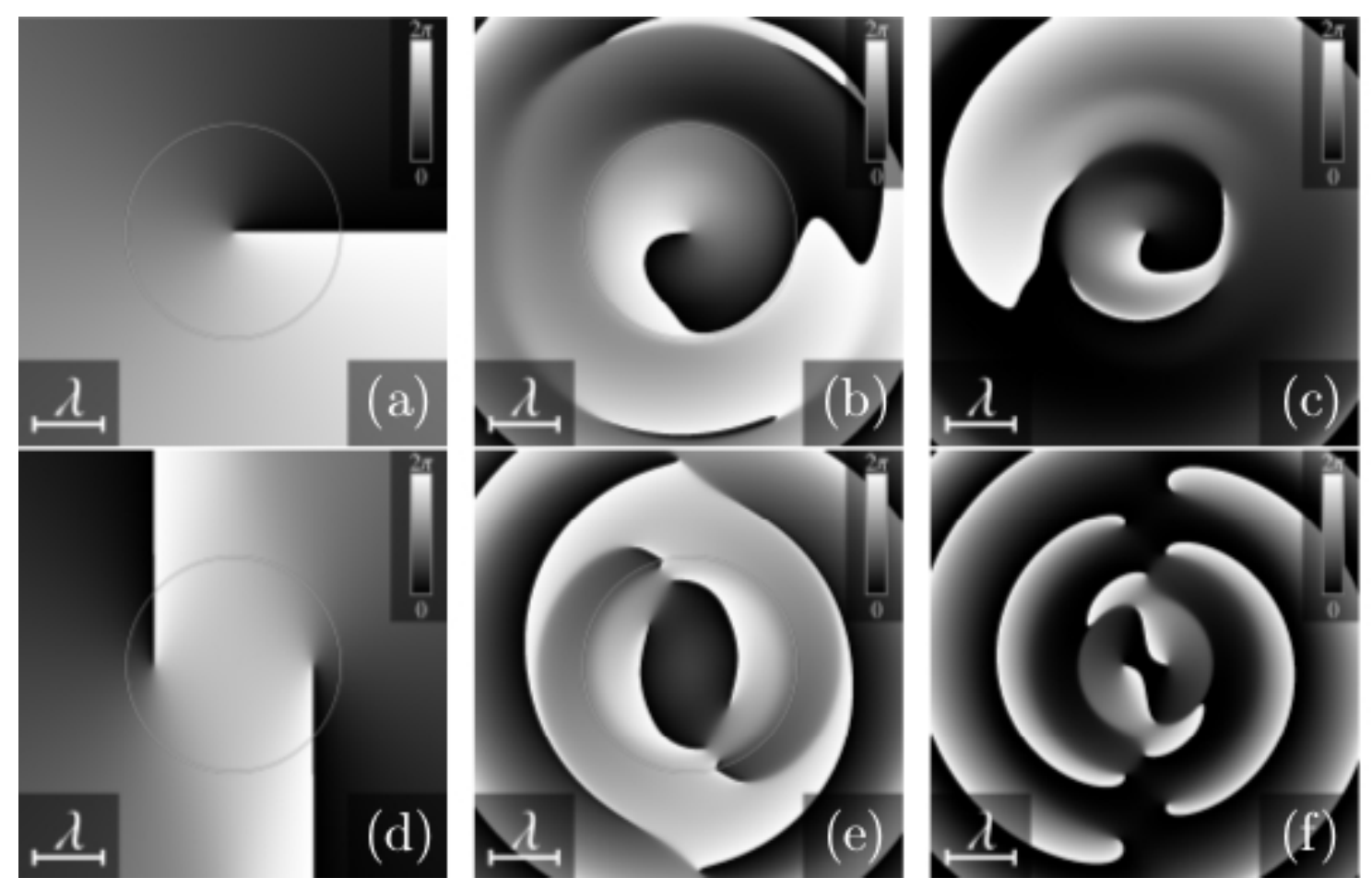

FIG. 7. Near-field phase maps of the electric field components $E_{x}$ (a,b,c) and $E_{z}$ (d,e,f) in the planes $z=0(\mathrm{a}, \mathrm{b}, \mathrm{d}, \mathrm{e})$ and $z=R_{p}=1.5 \lambda(\mathrm{c}, \mathrm{f})$ for the LG beam with $m_{\mathrm{LG}}=1$ and $f=0.1$. (a) [(d)] Phase map of the electric field component $E_{x}^{(\mathrm{LG})}$ $\left[E_{z}^{(\mathrm{LG})}\right]$ of the incident wave beam in the $x-y$ plane $(z=0)$. (b,c) $[(\mathrm{e}, \mathrm{f})]$ Phase maps for the electric field component $E_{x}\left[E_{z}\right]$ of the total light wavefield in the planes $z=0$ and $z=R_{p}$, respectively. 
Now we return to the optical vortex structure for the components $E_{z}$ and $E_{x}$. The $\phi$ dependence of $E_{z}$ can be written in the following form:

$$
\begin{aligned}
& \exp \left[-i m_{\mathrm{LG}} \phi\right] E_{z}=\exp \left[-i m_{\mathrm{LG}} \phi+i \chi_{z}\right]\left|E_{z}\right|=E_{+1}^{(z)} \exp [i \phi]+E_{-1}^{(z)} \exp [-i \phi]= \\
& \exp \left[i \psi_{+}^{(z)}\right]\left\{\left|E_{+1}^{(z)}\right| \exp \left[i\left(\phi+\psi_{-}^{(z)}\right)\right]+\left|E_{-1}^{(z)}\right| \exp \left[-i\left(\phi+\psi_{-}^{(z)}\right)\right]\right\},
\end{aligned}
$$

where $E_{ \pm 1}^{(z)}=\left(\mathbf{E}_{ \pm 1} \cdot \hat{\mathbf{z}}\right), 2 \psi_{ \pm}^{(z)}=\arg \left(E_{+1}^{(z)}\right) \pm \arg \left(E_{-1}^{(z)}\right)$ and $\chi_{z}$ is the phase of $E_{z}$.

The complex plane formula (69) describes an ellipse parametrized by the azimuthal angle $\phi$. It is centered at the origin with the major (minor) semiaxis of the length $E_{+}^{(z)}(R)$ $\left(\left|E_{-}^{(z)}(R)\right|\right)$, where $E_{ \pm}^{(z)}(R)=\left|E_{+1}^{(z)}(R)\right| \pm\left|E_{-1}^{(z)}(R)\right| R$ is the radius of circle $C_{R}$ in the plane of observation, $z=z_{0}$. Then the closed loop contour integral of the wave phase $\chi_{z}$ is:

$$
\begin{aligned}
& m_{z}=\frac{1}{2 \pi} \oint_{C_{R}} \mathrm{~d} \chi_{z}=m_{\mathrm{LG}}+\mu_{z}(R), \\
& \mu_{z}(R)=\operatorname{sign}\left(E_{-}^{(z)}(R)\right)=\operatorname{sign}\left(\left|E_{+1}^{(z)}(R)\right|-\left|E_{-1}^{(z)}(R)\right|\right) .
\end{aligned}
$$
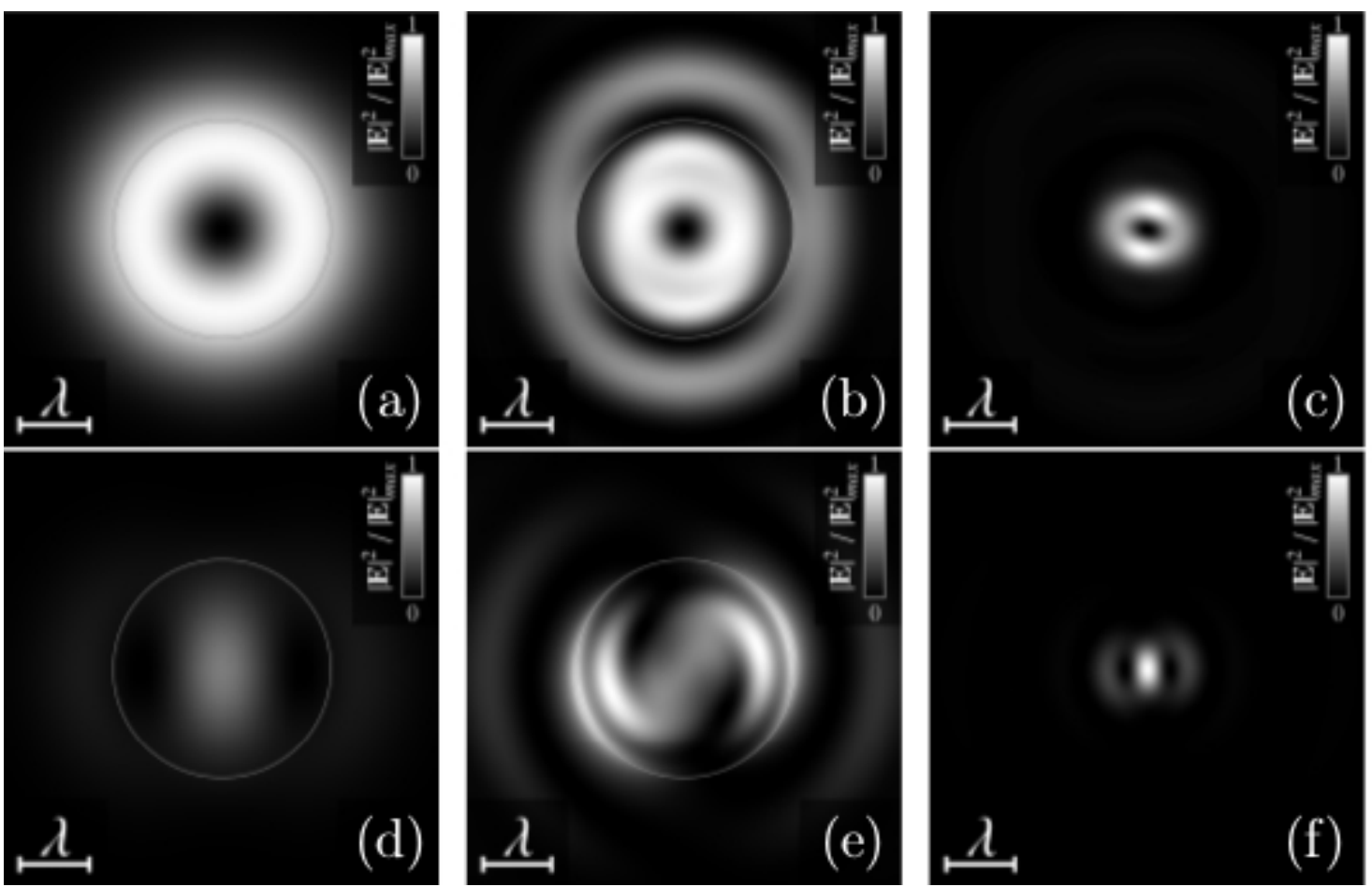

FIG. 8. Near-field intensity maps of the electric field components $\left|E_{x}\right|^{2}$ (a,b,c) and $\left|E_{z}\right|^{2}(\mathrm{~d}, \mathrm{e}, \mathrm{f})$ in the planes $z=0(\mathrm{a}, \mathrm{b}, \mathrm{d}, \mathrm{e})$ and $z=R_{p}=1.5 \lambda(\mathrm{c}, \mathrm{f})$ for the LG beam with $m_{\mathrm{LG}}=1$ and $f=0.1$. (a) [(d)] Intensity distribution for the $x$ [z] component, $\left|E_{x}^{(\mathrm{LG})}\right|^{2}\left[\left|E_{z}^{(\mathrm{LG})}\right|^{2}\right]$, of the incident wave beam in the $x-y$ plane $(z=0) .(\mathrm{b}, \mathrm{c})[(\mathrm{e}, \mathrm{f})]$ Intensity distributions for the $x[z]$ component of electric field of the total light wavefield in the planes $z=0$ and $z=R_{p}$, respectively.

From Eq. (70), the net topological charge of vortices encircled by $C_{R}$ can be either $m_{\mathrm{LG}}+1$ or $m_{\mathrm{LG}}-1$. At $\left|E_{+1}^{(z)}(R)\right|=\left|E_{-1}^{(z)}(R)\right|, \mu_{z}(R)$ is undefined. This is the special case when $\left|E_{z}\right|=0$ at $\cos \left(\phi+\psi_{-}^{(z)}\right)=0$ and the circle contains a pair of symmetrically located vortices. Each of these vortices carries a charge of the magnitude equal to unity. Generally, 
the vortices are of the same sign, which is determined by the change of $\mu_{z}(R)$ as the radius $R$ passes a critical value. When $\mu_{z}(R)$ changes from $+1(-1)$ to $-1(+1)$ two vortices of the charge $-1(+1)$ intersect the boundary and move into the interior part of the circle.

The case of the $x$ component of the electric field, $E_{x}$, can be analyzed along similar lines. From Eq. (67), we deduce the $\phi$ dependence of $E_{x}$ in the form:

$$
\begin{aligned}
& \exp \left[-i m_{\mathrm{LG}} \phi+i \chi_{x}\right]\left|E_{x}\right|-E_{0}^{(x)}=E_{+2}^{(x)} \exp [2 i \phi]+E_{-2}^{(x)} \exp [-2 i \phi]= \\
& \exp \left[i \psi_{+}^{(x)}\right]\left\{\left|E_{+2}^{(x)}\right| \exp \left[i\left(2 \phi+\psi_{-}^{(x)}\right)\right]+\left|E_{-2}^{(x)}\right| \exp \left[-i\left(2 \phi+\psi_{-}^{(x)}\right)\right]\right\},
\end{aligned}
$$

where $E_{ \pm 2,0}^{(x)}=\left(\mathbf{E}_{ \pm 2,0} \cdot \hat{\mathbf{x}}\right), 2 \psi_{ \pm}^{(x)}=\arg \left(E_{+2}^{(x)}\right) \pm \arg \left(E_{-2}^{(x)}\right)$ and $\chi_{x}$ is the phase of $E_{x}$. The center of the ellipse described by Eq. (71) is generally displaced from the origin and is determined by $E_{0}^{(x)}$. The length of its major (minor) semiaxis is $E_{+}^{(x)}(R)\left(\left|E_{-}^{(x)}(R)\right|\right)$, where $E_{ \pm}^{(x)}(R)=$ $\left|E_{+2}^{(x)}(R)\right| \pm\left|E_{-2}^{(x)}(R)\right|$.

The closed loop contour integral of the wave phase $\chi_{x}$ is:

$$
m_{x}=\frac{1}{2 \pi} \oint_{C_{R}} \mathrm{~d} \chi_{x}=m_{\mathrm{LG}}+\mu_{x}(R), \quad \mu_{x}(R) \in\{-2,0,2\} .
$$
relation:

When the origin is enclosed by the ellipse (71), similar to Eq. (70b), we have the

$$
\mu_{x}(R)=2 \operatorname{sign}\left(E_{-}^{(x)}(R)\right)=2 \operatorname{sign}\left(\left|E_{+2}^{(x)}(R)\right|-\left|E_{-2}^{(x)}(R)\right|\right) .
$$

In the opposite case, when the origin is outside the area encircled by the ellipse, $\mu_{x}(R)$ is zero. The latter is the case for the near-field phase maps shown in Figs. 7(a)-(c) that represent the 2D distributions of $\chi_{x}$ in the $x-y$ plane for the LG beam with $m_{\mathrm{L} G}=1$ (see Fig. 2).

As is evident from Figs. 7(a)-(c) (see also the intensity maps in Figs. 8(a)-(c)), in these distributions, the only vortex is positioned at the center and possesses the charge $m_{x}=m_{\mathrm{LG}}=$ +1 . As opposed to the case with $m_{\mathrm{LG}}=2$ discussed in Ref. [38], at $m_{\mathrm{LG}}=1$, the central vortex of the $x$ component, $E_{x}$, is structurally stable and cannot be destroyed.

The near-field phase maps for $\chi_{z}$ are presented in Figs. 7(d)-(f). Figure 7(d) shows the $2 \mathrm{D}$ map for the incident optical vortex LG beam with $m_{\mathrm{LG}}=1$ in the focal plane $z=0$. The corresponding intensity map is depicted in Fig. 8(d). It is seen that there are no vortices at the center, so that, at sufficiently small $R, m_{z}=0$ and $\mu_{z}=-1$. In addition, there is a pair of the symmetrically-arranged vortices of the charge +1 inside the particle. So, when the radius $R$ is large enough for the circle to enclose the vortices, the total charge is $m_{z}=m_{\mathrm{LG}}+1=2$ and $\mu_{z}=1$.

For the total wavefield at $z=0$, the phase and intensity maps are given in Fig. 7(e) and Fig. 8(e), respectively. It can be seen that the vortex pattern is complicated by interference between the incident and the scattered waves. Referring to Fig. 7(e), there are two additional pairs of vortices whose charges are opposite in sign. The negatively charged vortices (the charge is -1 ) are located inside the particle, whereas the positively charged ones (the charge is +1 ) are formed on the surface of the particle. A similar structure is discernible from Figs. 7(f) and 8(f), representing the results for the plane tangent to the particle surface $z=R_{p}$.

\section{Conclusions}

In this paper, we have used a $T$-matrix approach in the form described in Refs. $[10,38]$ to study the light scattering problem for optically isotropic spherical scatterers illuminated with LG beams that represent optical vortex laser beams. Our approach uses the remodelling procedure 
in which the far-field matching method is combined with the results for nonparaxial propagation of LG beams. Scattering of such beams is thus described in terms of the far-field angular distributions, $\mathbf{E}_{\text {out }}^{\text {(inc) }}$ and $\mathbf{E}_{\text {out }}^{\text {(sca) }}$, that determine the outgoing parts of the incident and scattered waves. The far-field distributions play a central role in the method giving, in particular, the differential cross-sections and the optical (radiation) force acting upon the Mie scatterer.

The analytical results are employed to perform numerical analysis of the optical field in the near-field region. In order to examine the effects of incident beam spatial structure on the light wavefield near the scatterer, we have computed a number of the $2 \mathrm{D}$ near-field intensity and phase distributions for purely azimuthal LG beams. In this case, a LG beam possesses the vanishing radial mode number and carries the optical vortex with the topological charge characterized by the azimuthal number $m_{\mathrm{LG}}$.

The 2D near-field intensity distributions computed for the plane-wave limiting case in which the incident wave is a Gaussian beam $\left(m_{\mathrm{LG}}=0\right)$ with small focusing parameter $f$ $\left(2 \pi f=\lambda / w_{0}<1\right)$ reveal the well-known structure of photonic nanojets (see Fig. 1). Figures 24 represent the results for the LG beams with $1 \leq m_{\mathrm{LG}} \leq 2$ and show that the morphology of photonic jets formed at $m_{\mathrm{LG}} \neq 0$ significantly differs from the well-known shape of nanojet at $m_{\mathrm{LG}}=0$. The effect that a jetlike photonic flux emerging from the particle shadow surface can be formed even if the bulk part of the scatterer is in the low intensity region is illustrated in Figs. 2(b)-3(b)). In contrast, as can be seen from Fig. 5, the jetlike flux near negative index metamaterial Mie scatterers may emerge from the illuminated part of the particle surface.

The form of the beam shape coefficients (65) is dictated by the twofold rotational symmetry of the LG beam (see Sec. 3.3) and underlies general formula (67) giving the electric field vector expressed as a function of the azimuthal angle $\phi$. The latter is at the heart of our analysis of optical vortices associated with the electric field components.

An important consequence of Eq. (67) is that, at sufficiently large azimuthal numbers, $\left|m_{\mathrm{LG}}\right| \geq 3$, light scattering of LG beams takes place without destroying the optical vortex located on the beam axis. By contrast, at $\left|m_{\mathrm{LG}}\right|<3$, the intensity of scattered wavefield does not vanish on the beam axis so that, in the near-field region, light scattering has a destructive effect on the optical vortex (see Figs. 2-4).

Using analytical expressions (69) and (71), we have described the geometry of optical vortices for the components $E_{z}$ and $E_{x}$ in the planes $z=z_{0}$ normal to the beam axis (the $z$ axis). It was found that, except for the central vortex, the topological charge of off-center vortices generally equals unity in magnitude. They are organized into pairs of symmetricallyarranged and equally-charged vortices. These pairs lie on concentric circles and their vortex charge alternate in sign with the circle radius (see, e.g., Fig. 7(f)).

The phase maps of $E_{x}$ shown in Figs. 7(a)-(e) (the corresponding square amplitude distributions are presented in Figs. 8(a)-(e)) are computed for the LG beam with $m_{\mathrm{LG}}=1$. The central vortex having azimuthal number $m_{\mathrm{LG}}=1$ was found to be the only vortex for both the incident beam and the total wavefield. Formula (69) implies that the $z$ axis is a nodal line for the $x$ component of the electric field, $E_{x}$, and the central vortex is structurally stable at $m_{\mathrm{LG}}=1$. When $m_{\mathrm{LG}}=2$, a similar result applies to the $z$ component [38].

In the phase maps for $E_{z}$, depicted in Fig. 7(d)-(f), there are no vortices at the origin. For the incident wave, there is a pair of equally charged vortices (see Fig. 7(d)). As is seen from Figs. 7(e)-(f), interference between the incident and the scattered waves produces additional pairs of symmetrically arranged vortices. 


\section{Acknowledgments}

A.D.K. acknowledges partial financial support from the Government of the Russian Federation (Grant No. 074-U01), from the Ministry of Education and Science of the Russian Federation (Grant No. GOSZADANIE 2014/190, Project No. 14.Z50.31.0031, and ZADANIE Grant No. 1.754.2014/K), through a grant from the Russian Foundation for Basic Research, and through a grant from the President of Russia (Grant No. MK-2736.2015.2).

\section{References}

[1] Mie G. Beiträge zur Optik trüber Medien, speziell kolloidaler Metallösungen. Ann. Phys., Leipzig, 1908, 25, P. $377-445$.

[2] Newton R. G. Scattering Theory of Waves and Particles. 2 edition. Heidelberg, Springer, 1982, 745 p.

[3] Tsang L., Kong J. A., Ding K.-H. Scattering of Electromagnetic Waves. Theories and Applications. NY : Wiley-Interscience Pub, 2000. Vol. 1 of Wiley Series in Remote Sensing. 426 p.

[4] Mishchenko M. I., Travis L. D., Lacis A. A. Scattering, Absorption and Emission of Light by Small Particles. NY : Cambridge University Press, 2004, 448 p.

[5] Gouesbet G., Gréhan G. Generalized Lorenz-Mie theories. Berlin, Springer, 2011, 310 p.

[6] Mishchenko M. I., Travis L. D., Mackowski D. W. T-matrix computations of light scattering by nonspherical particles: a review. J. of Quant. Spectr., \& Radiat. Transf., 1996, 55, P. 535-575.

[7] Light Scattering by Nonspherical Particles: Theory, Measurements and Applications / Ed. by M. I. Mishchenko, J. W. Hovenier, L. D. Travis. New York, Academic Press, 2000.

[8] Roth J., Digman M. J. Scattering and extinction cross sections for a spherical particle coated with an oriented molecular layer. J. Opt. Soc. Am., 1973, 63, P. 308-311.

[9] Kiselev A. D., Reshetnyak V. Y., Sluckin T. J. Influence of the optical axis distribution in the anisotropic layer surrounding a spherical particle on the scattering of light. Opt. Spectrosc., 2000, 89(6), P. 907-913.

[10] Kiselev A. D., Reshetnyak V. Y., Sluckin T. J. Light scattering by optically anisotropic scatterers: $T$-matrix theory for radial and uniform anisotropies. Phys. Rev. E, 2002, 65(5), P. 056609.

[11] Geng Y.-L., Wu X.-B., Li L.-W., Guan B.-R. Mie scattering by a uniaxial anisotropic sphere. Phys. Rev. E, 2004, 70, P. 056609.

[12] Novitsky A., Barkovsky L. Matrix approach for light scattering from a multilayered rotationally symmetric bianisotropic sphere. Phys. Rev. A, 2008, 77, P. 033849.

[13] Chengwei Qiu, Lei Gao, John D. Joannopoulos, Marin Soljačić. Light scattering from anisotropic particles: propagation, localization and nonlinearity. Laser \& Photon. Rev., 2010, 4(2), P. 268-282.

[14] Grehan G., Maheu B., Gouesbet G. Scattering of laser beams by Mie scatter centers: numerical results using a localized approximation. Appl. Opt., 1986, 25(19), P. 3539-3548.

[15] Gouesbet G., Maheu B., Gréhan G. Light scattering from a sphere arbitrarily located in a Gaussian beam, using a Bromwich formulation. J. Opt. Soc. Am. A, 1988, 5(9), P. 1427-1443.

[16] Barton J. P., Alexander D. R., Schaub S. A. Internal and near-surface electromagnetic fields for a spherical particle irradiated by a focused laser beam. J. Appl. Phys., 1988, 64(4), P. 1632-1639.

[17] Barton J. P., Alexander D. R., Schaub S. A. Theoretical determination of net radiation force and torque for a spherical particle illuminated by a focused laser beam. J. Appl. Phys., 1989, 66(10), P. 4594-4602.

[18] Schaub S. A., Alexander D. R., Barton J. P. Glare spot image calculations for a spherical particle illuminated by a tightly focused beam. J. Opt. Soc. Am. A, 1992, 9(2), P. 316-330.

[19] Lock J. A., Gouesbet G. Generalized Lorenz-Mie theory and applications. J. of Quant. Spectr. \& Radiat. Transf., 2009, 110, P. 800-807.

[20] Gouesbet G., Lock J. A., Gréhan G. Generalized Lorenz-Mie theories and description of electromagnetic arbitrary shaped beams: Localized approximations and localized beam models, a review. J. of Quant. Spectr. \& Radiat. Transf., 2011, 112, P. 1-27.

[21] Lax M., Louisell W. H., McKnight W. B. From Maxwell to paraxial wave optics. Phys. Rev. A, 1975, 11(4), P. $1365-1370$.

[22] Davis L. W. Theory of electromagnetic waves. Phys. Rev. A, 1979, 19, P. 1177-1179.

[23] Nieminen T. A., Rubinsztein-Dunlop H., Heckenberg N. R. Multipole expansion of strongly focussed laser beams. J. of Quant. Spectr. \& Radiat. Transf., 2003, 79-80, P. 1005-1017.

[24] Bareil P. B., Sheng Y. Modeling highly focused laser beam in optical tweezers with the vector Gaussian beam in the T-matrix method. J. Opt. Soc. Am. A, 2013, 30(1), P. 1-6. 
[25] Hoang T. X., Chen X., Sheppard C. J. R. Multipole theory for tight focusing of polarized light, including radially polarized and other special cases. J. Opt. Soc. Am. A, 2012, 29(1), P. 32-43.

[26] Barnett S. M., Allen L. Orbital angular momentum and nonparaxial light beams. Opt. Commun., 1994, 110(5-6), P. 670-678.

[27] Duan K., Wang B., Lü B. Propagation of Hermite-Gaussian and Laguerre-Gaussian beams beyond the paraxial approximation. J. Opt. Soc. Am. A, 2005, 22(9), P. 1976-1980.

[28] Van De Nes A. S., Pereira S. F., Braat J. J. M. On the conservation of fundamental optical quantities in non-paraxial imaging systems. Journal of Modern Optics, 2006, 53(5-6), P. 677-687.

[29] Zhou G. Analytical vectorial structure of Laguerre-Gaussian beam in the far field. Opt. Lett., 2006, 31(17), P. 2616.

[30] Zhou G. Propagation of a vectorial Laguerre-Gaussian beam beyond the paraxial approximation. Optics \& Laser Technology, 2008, 40(7), P. 930-935.

[31] van de Nes A. S., Török P. Rigorous analysis of spheres in Gauss-Laguerre beams. Opt. Express, 2007, 15(20), P. 13360-13374.

[32] Yuesong Jiang, Yuwei Shao, Xiaosheng Qu et al. Scattering of a focused Laguerre-Gaussian beam by a spheroidal particle. J. Opt., 2012, 14(12), P. 125709.

[33] Desyatnikov A. S., Kivshar Y. S., Torner L. Optical vortices and vortex solitons. Progress in Optics / Ed. by E. Wolf. Amsterdam, North-Holland, 2005. Vol. 47. P. 291-391.

[34] Optical Angular Momentum / Ed. by L. Allen, S. M. Barnett, M. J. Padgett. London, Taylor \& Francis, 2003.

[35] Structured Light and Its Applications: An Introduction to Phase-Structured Beams and Nanoscale Optical Forces / Ed. by David L. Andrews. Amsterdam, Academic Press, 2008. 342 p.

[36] Simpson S. H., Hanna S. Rotation of absorbing spheres in Laguerre-Gaussian beams. J. Opt. Soc. Am. A, 2009, 26(1), P. 173-183.

[37] Simpson S. H., Hanna S. Orbital motion of optically trapped particles in Laguerre-Gaussian beams. J. Opt. Soc. Am. A, 2010, 27(9), P. 2061-2071.

[38] Kiselev A. D., Plutenko D. O. Mie scattering of Laguerre-Gaussian beams: Photonic nanojets and near-field optical vortices. Phys. Rev. A, 2014, 89, P. 043803. http://dx.doi.org/10.1103/PhysRevA.89.04380

[39] A. Heifetz, Soon-Cheol Kong, A. V. Sahakian et al. Photonic Nanojets. J. Comput. Theor. Nanosci., 2009, 12(7), P. 1214-1220.

[40] Chen Z., Taflove A., Backman V. Photonic nanojet enhancement of backscattering of light by nanoparticles: a potential novel visible light ultramicroscopy technique. Opt. Express, 2004, 12(7), P. 1214-1220.

[41] Xu Li, Zhigang Chen, Allen Taflove, Vadim Backman. Optical analysis of nanoparticles via enhanced backscattering facilitated by 3-D photonic nanojets. Opt. Express, 2005, 13(2), P. 526-533.

[42] Biedenharn L. C., Louck J. D. Angular Momentum in Quantum Physics: Theory and Application. Reading, Massachusetts, Addison-Wesley, 1981. Vol. 8 of Encyclopedia of Mathematics and its Applications. 717 p.

[43] Varshalovich D. A., Moskalev A. N., Khersonskii V. K. Quantum theory of angular momentum: Irreducible tensors, spherical harmonics, vector coupling coefficients, 3nj symbols. Singapore, World Scientific Publishing Co., 1988. 514 p.

[44] Jackson J. D. Classical Electrodynamics, 3rd edition. New York, Wiley, 1999.

[45] Sarkar D., Halas N. J. General vector basis function solution of Maxwells equations. Phys. Rev. E, 1997, 56(1), P. 1102-1112.

[46] Handbook of Mathematical Functions / Ed. by M. Abramowitz, I. A. Stegun. New York, Dover, 1972.

[47] Kiselev A. D., Reshetnyak V. Y., Sluckin T. J. T-matrix theory of light scattering by uniformly anisotropic scatterers. Mol. Cryst. Liq. Cryst., 2002, 375, P. 373-386.

[48] Stout B., Neviére M., Popov E. Mie scattering by an anisotropic object. Part I. Homogeneous sphere. J. Opt. Soc. Am. A, 2006, 23 (5), P. 1111-1123.

[49] Gradshteyn I. S., Ryzhik I. M. Table of Integrals, Series, and Products. New York, Academic, 1980.

[50] Sherman G. C., Stamnes J. J., Lalor E. Asymptotic approximations to angular-spectrum representations. J. Math. Phys., 1976, 17(5), P. 760-776.

[51] Simpson S. H., Hanna S. First-order nonconservative motion of optically trapped nonspherical particles. Phys. Rev. E, 2010, 82, P. 031141.

[52] Rury A. S., Freeling R. Mie scattering of purely azimuthal Laguerre-Gauss beams: Angular-momentuminduced transparency. Phys. Rev. A, 2012, 86, P. 053830.

[53] Lecler S., Takakura Y., Meyrueis P. Properties of a three-dimensional photonic jet. Opt. Lett., 2005, 30(19), P. 2641-2643. 
[54] Devilez A., Stout B., Bonod N., Popov E. Spectral analysis of three-dimensional photonic jets. Opt. Express, 2008, 16(18), P. 14200-14212.

[55] Geints Y. E., Zemlyanov A. A., Panina E. K. Control over parameters of photonic nanojets of dielectric microspheres. Opt. Commun., 2010, 283(23), P. 4775-4781.

[56] Devilez A., Bonod N., Wegner J., et al. Three-dimensional subwavelength confinement of light with dielectric microspheres. Opt. Express, 2009, 17(4), P. 2089-2094.

[57] Myun-Sik Kim, Toralf Scharf, Stefan Mühlig et al. Engineering photonic nanojets. Opt. Express, 2011, 19(11), P. 10206-10220.

[58] Solymar L., Shamonina E. Waves in Metamaterials. NY, Oxford University Press, 2009. 385 p.

[59] Cai W., Shalaev V. Optical Metamaterials. Fundamentals and Applications, NY, Springer, 2010.200 p.

[60] Kivshar Y. S. From metamaterials to metasurfaces and metadevices. Nanosystems: Physics, Chemistry, Mathematics, 2015, 6(3), P. 346-352.

[61] Pravdin K. V., Popov I. Y. Photonic crystal with negative index material layers. Nanosystems: Physics, Chemistry, Mathematics, 2014, 5(5), P. 626-643.

[62] Veselago V. G. The electrodynamics of substances with simultaneously negative values of $\epsilon$ and $\mu$. Soviet Physics Uspekhi, 1968, 10(4), P. 509-514. 